\title{
Spatially-resolved star formation histories of CALIFA galaxies
}

\section{Implications for galaxy formation}

\author{
R. M. González Delgado ${ }^{1}$, E. Pérez ${ }^{1}$, R. Cid Fernandes ${ }^{2}$, R. García-Benito ${ }^{1}$, R. López Fernández ${ }^{1}$, N. Vale Asari ${ }^{2}$, \\ C. Cortijo-Ferrero ${ }^{1}$, A. L. de Amorim² ${ }^{2}$ E. A. D. Lacerda ${ }^{2}$, S. F. Sánchez ${ }^{3}$, M. D. Lehnert ${ }^{4}$, and C. J. Walcher ${ }^{5}$ \\ 1 Instituto de Astrofísica de Andalucía (CSIC), PO Box 3004, 18080 Granada, Spain \\ e-mail: rosa@iaa.es \\ 2 Departamento de Física, Universidade Federal de Santa Catarina, PO Box 476, 88040-900 Florianópolis, SC, Brazil \\ 3 Instituto de Astronomía,Universidad Nacional Autonóma de México, A.P. 70-264, 04510 México D.F., Mexico \\ 4 Institut d'Astrophysique de Paris, UMR 7095, CNRS, Université Pierre et Marie Curie, 98 bis boulevard Arago, 75014 Paris, France \\ 5 Leibniz-Institut für Astrophysik Potsdam (AIP), An der Sternwarte 16, 14482 Potsdam, Germany
}

Received 28 March 2017 / Accepted 13 June 2017

\begin{abstract}
This paper presents the spatially resolved star formation history (SFH) of nearby galaxies with the aim of furthering our understanding of the different processes involved in the formation and evolution of galaxies. To this end, we apply the fossil record method of stellar population synthesis to a rich and diverse data set of 436 galaxies observed with integral field spectroscopy in the CALIFA survey. The sample covers a wide range of Hubble types, with stellar masses ranging from $M_{\star} \sim 10^{9}$ to $7 \times 10^{11} M_{\odot}$. Spectral synthesis techniques are applied to the datacubes to retrieve the spatially resolved time evolution of the star formation rate (SFR), its intensity $\left(\Sigma_{\mathrm{SFR}}\right)$, and other descriptors of the 2D SFH in seven bins of galaxy morphology (E, S0, Sa, Sb, Sbc, Sc, and Sd) and five bins of stellar mass. Our main results are that (a) galaxies form very fast independently of their current stellar mass, with the peak of star formation at high redshift $(z>2)$. Subsequent star formation is driven by $M_{\star}$ and morphology, with less massive and later type spirals showing more prolonged periods of star formation. (b) At any epoch in the past, the SFR is proportional to $M_{\star}$, with most massive galaxies having the highest absolute (but lowest specific) SFRs. (c) While today, the $\Sigma_{\mathrm{SFR}}$ is similar for all spirals and significantly lower in early-type galaxies (ETG), in the past, the $\Sigma_{\mathrm{SFR}}$ scales well with morphology. The central regions of today's ETGs are where the $\Sigma_{\text {SFR }}$ reached the highest values $\left(>10^{3} M_{\odot} \mathrm{Gyr}^{-1} \mathrm{pc}^{-2}\right.$ ), similar to those measured in high-redshift star-forming galaxies. (d) The evolution of $\Sigma_{\mathrm{SFR}}$ in Sbc systems matches that of models for Milky Way-like galaxies, suggesting that the formation of a thick disk may be a common phase in spirals at early epochs. (e) The SFR and $\Sigma_{\text {SFR }}$ in outer regions of E and S0 galaxies show that they have undergone an extended phase of growth in mass between $z=2$ and 0.4. The mass assembled in this phase is in agreement with the two-phase scenario proposed for the formation of ETGs. (f) Evidence of an early and fast quenching is found only in the most massive $\left(M_{\star}>2 \times 10^{11} M_{\odot}\right)$ E galaxies of the sample, but not in spirals of similar mass, suggesting that halo quenching is not the main mechanism for the shut down of star formation in galaxies. Less massive E and disk galaxies show more extended SFHs and a slow quenching. (g) Evidence of fast quenching is also found in the nuclei of ETG and early spirals, with SFR and $\Sigma_{\text {SFR }}$ indicating that they can be the relic of the "red nuggets" detected at high redshift.
\end{abstract}

Key words. galaxies: evolution - Galaxy: stellar content

\section{Introduction}

Our understanding of how galaxies form has undergone significant progress in recent years that is due to improvements in observational facilities and the diversity of techniques with which galaxy properties are estimated. A large number of surveys, performed at a range of redshifts that sample the age of the Universe, have allowed us to establish relations of the distribution and the properties of galaxies with the complex distribution of dark matter halos, filaments, and voids that form the cosmic web. However, the main driver for galaxy assembly is still unknown at present; accretion and merging are the two most important mechanisms that have been proposed.

On the theoretical front, the $\Lambda$ cold dark matter paradigm poses that galaxies grow their mass by merging of dark matter halos, progressively assembling more massive systems by bringing stars from subsystems with different histories to what eventually becomes a single massive galaxy. Simulations suggest that major and minor mergers make up as much as $50 \%$ of the outer envelopes of massive galaxies (Naab et al. 2009), but observations indicate that equal-mass mergers are rare and relatively unimportant for the cosmic star formation budget (Man et al. 2012; Williams et al. 2011). Moreover, there are also difficulties in matching the number of thin-disk galaxies in this scenario (Naab \& Ostriker 2016).

Galaxies with stellar masses $M_{\star} \sim 10^{11} M_{\odot}$ mark the transition between galaxies that are dominated by in situ star formation growth (at low mass) and those dominated by merger growth (at high mass; Behroozi et al. 2013). Milky Way (MW) like galaxies and those of lower mass seem to have assembled their mass through streams of cold gas from the cosmic web (Sánchez Almeida et al. 2014). In this context, the gas accretion and star formation rates (SFR) of the galaxy are expected to be associated with the specific accretion rate of the cosmological dark matter (Neistein et al. 2006; Birnboim et al. 2007; Neistein \& Dekel 2008; Dutton et al. 2010). 
Observationally, several fundamental results related to how galaxies grow their stellar mass are now well established: (1) the SFR density in the Universe peaked 10 Gyr ago, at redshift $z \sim 2$, and declined thereafter (Lilly et al. 1996; Madau et al. 1998; Hopkins \& Beacom 2006; Fardal et al. 2007; Madau \& Dickinson 2014). (2) At any $z$, star-forming galaxies show a correlation between SFR and $M_{\star}$, known as the main sequence of star formation (MSSF; Brinchmann et al. 2004; Noeske et al. 2007; Daddi et al. 2007; Elbaz et al. 2007; Wuyts et al. 2011; Whitaker et al. 2012; Renzini \& Peng 2015; Catalán-Torrecilla et al. 2015; Cano-Díaz et al. 2016). (3) The specific star formation rate, $s S F R=S F R / M_{\star}$, declines weakly with increasing galaxy mass (Salim et al. 2007; Schiminovich et al. 2007), evolving rapidly at $z<2$ (Rodighiero et al. 2010; Oliver et al. 2010; Karim et al. 2011; Elbaz et al. 2011; Speagle et al. 2014), and increasing slowly at $z>2$ (Magdis et al. 2010; Stark et al. 2013). (4) Galaxy colors, morphology, chemical composition, and spectral type all show bimodal distributions, with blue star-forming galaxies separated from red quiescent galaxies (Blanton et al. 2003; Kauffmann et al. 2003; Baldry et al. 2004; Gallazzi et al. 2005; Mateus et al. 2006; Blanton \& Moustakas 2009). This bimodality is also intrinsic in the Hubble classification of galaxies (e.g., Roberts \& Haynes 1994; Kennicutt 1998). (5) Galaxies in the blue cloud must evolve to the red sequence, since the population of local quiescent galaxies is not consistent with a simple passive evolution of the population of quiescent galaxies at $z \sim 1$ (Faber et al. 2007; Bell et al. 2007; Gallazzi et al. 2014).

These results suggest that the growth of galaxies is not driven solely by gas supply; other physical processes, such as feedback from supernovae, are required to decouple the star formation from the gas accretion (Dekel et al. 2009; Lilly et al. 2013). For example, Lehnert et al. (2014) proposed a two-phase scenario to explain the evolution of the SSFR: (1) at $z>2$, the star formation is self-regulated by starburst-driven outflows. (2) At $z<2$, the decline of the sSFR is driven not only by declining gas fractions, but also by the evolution in the angular momentum to support the accreting gas to the disk, and by gas density and stellar mass surface density relations through a generalized Schmidt-Kennicut law (Dopita \& Ryder 1994; Shi et al. 2011). Furthermore, feedback from active galactic nucleus (AGN) can cause the suppression of gas accretion onto galaxies by heating the surrounding gas (Di Matteo et al. 2005).

Alternative mechanisms to feedback (from stars or AGN) have been proposed to explain the complete cessation of star formation and the end of the growth of massive galaxies. In the halo-quenching scenario, circumgalactic gas becomes shockheated above a critical halo mass of $\sim 10^{12} M_{\odot}$ and stops cooling (Dekel \& Birnboim 2006; Croton et al. 2006). The morphological transformation by the formation of a spheroidal component, known as morphological quenching, can also stop the star formation by stabilizing the gas disk against fragmentation (Martig et al. 2009; Genzel et al. 2014; González Delgado et al. 2015; Belfiore et al. 2017). Thanks to spatial resolution information on the SSFR, it has recently been found that this quenching progresses inside-out (Tacchella et al. 2015, 2016; González Delgado et al. 2015, 2016).

In these scenarios the growth of galaxies is a uniform phenomenon that is interrupted by internal or external quenching processes. However, alternative views are emerging in which galaxy growth is a more heterogeneous phenomenon, as suggested by the diversity of star formation histories (SFH) obtained in several surveys (Gladders et al. 2013; Oemler et al. 2013; Dressler et al. 2016; Abramson et al. 2016). These works show that the SFH may be extended or compressed in time (Asari et al. 2007; Pacifici et al. 2016).

Simple analytic models for the SFH have been explored for decades to infer the SFR (and sSFR) of galaxies at different redshifts by fitting their spectral energy distributions. However, parametrizations like an exponential declining function or the so-called delayed $\tau$ models (Searle et al. 1973; Bruzual A. \& Kron 1980) are not able to predict the rising epoch of the SFH of the Universe. Models that assume a rising SFH have been more successful to explain the evolution of the SFR at $z>2$ (e.g., Papovich et al. 2011; Maraston et al. 2013; Behroozi et al. 2013). A lognormal function has been proposed by Gladders et al. (2013) to represent both the cosmic evolution of the SFR of the Universe and the diversity of SFHs observed in individual galaxies. Furthermore, the effect that the SFH of galaxies have on the MSSF relation (Cassarà et al. 2016) or how galaxies stop their mass growth has just started to be explored (Pacifici et al. 2016).

Alternatively to redshift studies, the SFH of galaxies can be inferred using the fossil record encoded in their presentday stellar populations. This technique has changed significantly since the seminal works by Tinsley $(1968,1972)$, Searle et al. (1973), Gallagher et al. (1984), and Sandage (1986), who first used optical colors to study how the SFHs of galaxies vary along the Hubble sequence. It has gained significantly with the development of full spectral synthesis and fitting codes where no a priori assumption is made about the functional form of the SFH (Panter et al. 2003, 2008; Heavens et al. 2004; Cid Fernandes et al. 2005; Ocvirk et al. 2006; Asari et al. 2007; Tojeiro et al. 2011; Koleva et al. 2011; McDermid et al. 2015; Citro et al. 2016).

Regardless of the method, most of the observational work reviewed above is based on spatially integrated data, where the different morphological components (bulge and disk) are not separated or the data only partially cover the extent of a galaxy.

Recently, a new generation of integral field spectroscopy (IFS) surveys has emerged to overcome these limitations, such as ATLAS3D (Cappellari et al. 2011), CALIFA (Sánchez et al. 2012, 2016; Husemann et al. 2013; García-Benito et al. 2015), SAMI (Bryant et al. 2015), and MaNGA (Bundy et al. 2015; Law et al. 2015). These surveys are important to understand how the spheroidal and disk components in a galaxy form and evolve, and what their relative contributions are to the SFH of the Universe. CALIFA is particularly well suited for such a study because (1) its large field of view fully covers the galaxies; (2) its spectral range and resolution allow us to apply full spectral fitting to retrieve the SFH; (3) it includes galaxies of all Hubble types (E, S0, and spirals from Sa to Sd) and stellar masses $\left(\sim 10^{9}\right.$ to $\left.10^{12} M_{\odot}\right)$; (4) its well-defined selection function allows for reliable volume-corrections, and thus for an extrapolation of the results to a cosmic context (Walcher et al. 2014; Bekeraitè et al. 2016; González Delgado et al. 2016).

In previous papers in this series, we have employed fossil record tools to CALIFA datacubes and obtained the results we list below.

1. The mass-assembly history of $\sim 100$ galaxies (Pérez et al. 2013). We find that galaxies grow inside-out, and that the signal of downsizing is spatially preserved, with both inner and outer regions growing faster for massive galaxies. This inside-out scenario has recently been confirmed with MaNGA data by Ibarra-Medel et al. (2016).

2. The radial distribution of stellar populations properties (age, metallicity, and extinction) and their gradients as a function 
of Hubble type and $M_{\star}$ (González Delgado et al. 2014b, 2015). We find that more massive galaxies are more compact, older, more metal rich, and less dusty, and that these trends are preserved with radial distance to the nucleus. The age gradients also confirm an inside-out formation for both earlyand late-type galaxies. These results are also supported from CALIFA data reported by Sánchez-Blázquez et al. (2014), and by the analysis of Zheng et al. (2017) of MaNGA data, although not by Goddard et al. (2017), who found positive age radial gradients in their analysis of MaNGA data for early-type galaxies (ETG).

3 . The local relations between the stellar mass surface density $\left(\mu_{\star}\right)$ and age (González Delgado et al. 2014b), and stellar metallicity (González Delgado et al. 2014a). These indicate that $\mu_{\star}$ regulates the star formation and chemical enrichment in disks, while in spheroids, $M_{\star}$ is a more important driver. The bimodal behavior of the stellar ages and $\mu_{\star}$ has been confirmed by Zibetti et al. (2017) in an independent analysis of CALIFA data.

4. The existence of a tight relation between $\mu_{\star}$ and the intensity of the star formation $\Sigma_{\mathrm{SFR}}$ (defined as the SFR per unit area), defining a local MSSF relation with a slope similar to the global slope between total SFR and $M_{\star}$ (González Delgado et al. 2016). This suggests that local processes are important in determining the star formation in disks, probably through a density dependence of the SFR law. A similar $\Sigma_{\mathrm{SFR}}-\mu_{\star}$ relation was derived by Cano-Díaz et al. (2016) using the spatial distribution of $\mathrm{H} \alpha$ in CALIFA disk galaxies.

5. The radial profile of the recent SSFR as a function of Hubble type (González Delgado et al. 2016). These profiles increase outward, with a steeper slope in the inner $R<1$ halflight radius (HLR). This behavior suggests that galaxies are quenched inside-out and that this process is faster in the bulge-dominated regions than in the disks. A similar result was found by Tacchella et al. (2015) through analyzing a sample of star-forming galaxies at $z \sim 2.2$. These results are interpreted as evidence of the morphological quenching that these galaxies experience in the growth of their central bulges.

The list above illustrates the potential of combining spatially resolved spectroscopy with stellar population analysis tools as a means to gather clues on the processes that shaped the presentday galaxy population. Even though these studies resolve galaxies in time $(t)$ and space $(R)$, so far in this series the radial axis has been explored in far greater detail than the temporal one. While radial profiles of physical properties were mapped in the full resolution allowed by the data, the temporal information was mostly condensed to mean stellar ages or to an assessment of the star formation activity in the very recent past. This approach is justified because these low-order moments of the SFH are naturally the most robust descriptors obtained from any stellar population analysis. Despite the uncertainties involved, there is evidently more information encoded in the age distribution that is inferred from our fossil record method, however.

In this paper we explore this information by examining the temporally and spatially resolved SFH of CALIFA galaxies as a function of Hubble type and $M_{\star}$. Different representations of the SFH are used, including the SFR, sSFR, and $\Sigma_{\text {SFR }}$ as a function of lookback time and for different radial regions. These are then averaged over bins in stellar mass and morphology to obtain a panoramic view of the growth of galaxies. The aim is for this approach to lead to useful insights into questions like those listed below.

1. What are the main epochs of star formation in galaxies, and how do they change in time, place, and intensity as a function of current mass and morphology?

2. Can we identify phases like the spheroid and thick- and thindisk formation that are envisaged in current galaxy formation scenarios?

3. Do merger or accretion of stars from smaller systems leave detectable imprints in the spatially resolved SFHs of galaxies?

4. How do SFHs derived from a fossil record analysis compare to those inferred from the snapshots of galaxy evolution obtained by studies at different redshifts?

An ulterior goal of this paper is to make theorists aware of the power and limitations of these new tools for studying galaxy evolution, and in this way help establishing meaningful and enlightening ways of testing their models.

This paper is organized as follows. Section 2 describes the observations and summarizes the properties of the galaxies analyzed here. In Sects. 3 and 4 we summarize our method for extracting the SFH and present the spatially resolved results. Section 5 examines the cosmic evolution of the mass fraction, SFR, sSFR, and $\Sigma_{\text {SFR }}$ of galaxies as a function of Hubble type and $M_{\star}$ for three spatial regions: within $R<0.5 \mathrm{HLR}$, outside 1.5 HLR, and for the whole galaxy. In Sect. 6 we discuss the results in relation to different paradigms for the growth of spirals and early-type galaxies. Section 7 reviews our main findings.

In this paper we adopt for the relation between lookback time and redshift, $t(z)$, a flat cosmology: $\Omega_{\mathrm{M}}=0.3, \Omega_{\Lambda}=0.7$, and $H_{0}=70 \mathrm{~km} \mathrm{~s}^{-1} \mathrm{Mpc}^{-1}$. The units of $M_{\star}$ are $M_{\odot}$ throughtout; henceforth, we do not specify them for the sake of clarity.

\section{Data and sample}

\subsection{Observations and data reduction}

The observations were carried out at the Calar Alto observatory (CAHA) with the $3.5 \mathrm{~m}$ telescope and the Potsdam MultiAperture Spectrometer (PMAS; Roth et al. 2005) in the PPaK mode (Verheijen et al. 2004). The PPaK is an integral field spectrograph with a field of view of $74^{\prime \prime} \times 64^{\prime \prime}$ and 382 fibers of 2.7" diameter each (Kelz et al. 2006). The galaxies analyzed here were observed with two spectral setups, using the gratings V500 and V1200, with spectral resolutions $\sim 6$ and $2.3 \AA$ (FWHM), respectively. To reduce the effects of vignetting on the data, we combined the observations in the two setups (COMBO data, in the jargon of CALIFA), covering the 3700-7300 range with the same resolution as the V500 and a spatial sampling of $1 \mathrm{arcsec} / \mathrm{spaxel}$. The data were calibrated with version V2.2 of the reduction pipeline Sánchez et al. (2016). We refer to Sánchez et al. (2012, 2016), Husemann et al. (2013), and García-Benito et al. (2015) for details on the observational strategy and data processing.

\subsection{Sample and morphological classification}

The galaxies are from the main and extended CALIFA samples published in the third and final COMBO data release (DR3) by Sánchez et al. (2016). The galaxies targeted are a random subset of the mother sample plus some additional galaxies from the extended sample (a heterogeneous set of 38 sources observed in 


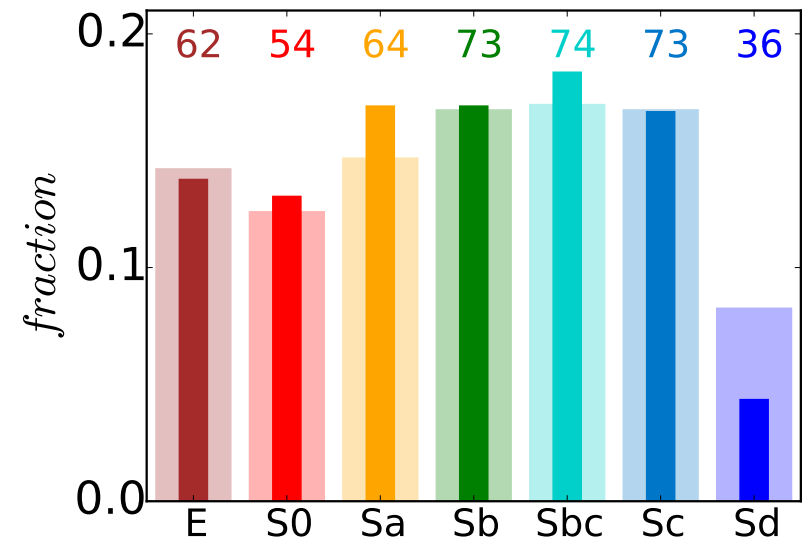

Fig. 1. Comparison of Hubble-type distributions in the CALIFA mother sample ( 939 galaxies, broad bars) and the 436 galaxies analyzed here (narrow, darker bars). The two histograms are normalized to unit sum. The number of galaxies in each morphology bin is labeled in color.

different ancillary projects). We further added 35 galaxies from the second data release that were previously analyzed by us in González Delgado et al. (2016). Type 1 Seyferts and galaxies that show merger or interaction features were excluded. This left a final sample of 436 galaxies, 398 of which belong to the mother sample. A full description and characterization of the mother sample is given by Walcher et al. (2014), and by Sánchez et al. (2016) for the extended sample. Briefly, they have (a) an angular isophotal diameter between 45" and 79"; (b) a redshift range $0.005 \leq z \leq 0.03$; and (c) colors $(u-r<5)$ and magnitudes $\left(-24<M_{r}<-17\right)$ that cover the whole color-magnitude diagram.

All galaxies were morphologically classified by members of the collaboration through visual inspection of the SDSS $r$-band images. As in previous works (González Delgado et al. 2015), we grouped the galaxies into seven morphology bins: E (62 galaxies), S0 (54, including S0 and S0a), Sa (64, including Sa and Sab), Sb (73), Sbc (74), Sc (73, including Sc and Scd), and $\mathrm{Sd}$ (36, including $1 \mathrm{Sm}$ and $1 \mathrm{Irr}$ ). Figure 1 shows the morphological distribution of our 436 galaxies (filled bars), and the distribution of the mother sample (wider bars). This distribution shows that this working sample is consistent with a random subset of the mother sample except for galaxies in the Sd-bin, which is undersampled in comparison with the others.

\section{Star formation history analysis}

\subsection{Analysis methods: STARLIGHT and PyCASSO}

The spatially resolved SFHs and related stellar population properties were extracted from the datacubes following the method originally presented in Pérez et al. (2013) and used in a series of works reviewed in Sect. 1. The method includes basic preprocessing steps, such as spatial masking of foreground and background sources, rest-framing, spectral resampling, extraction of individual spectra, and their stacking into Vorono zones (Cappellari \& Copin 2003) when it is necessary to reach a signal-to-noise ratio $S / N \geq 20$ (measured in a $90 \AA$ window centered at $5635 \AA$ rest-frame). Each spectrum was then fit with STARLIGHT (Cid Fernandes et al. 2005), which decomposes the spectra in terms of stellar population with ages $t_{j}$ and metallicities $Z_{j}$. The code outputs a population vector $\boldsymbol{x}$ whose components $x_{j}$ express the fractional contribution of base component $j$ to the observed continuum at a reference wavelength of $5635 \AA$. The corresponding mass fractions $\left(m_{j}\right)$ are also given.
The results were then processed through the Python CALIFA STARLIGHT Synthesis Organizer (PyCASSO; Cid Fernandes et al. 2013; de Amorim et al. 2017) to produce a suite of spatially resolved stellar population properties. PyCASSO organizes the information into datacubes with spatial as well as age and metallicity dimensions. Two of the main properties products used in this paper are the images of luminosity (at $5635 \AA$ ) per unit area, $\mathcal{L}_{\lambda 5635}$ (in units of $L_{\odot} \AA^{-1} \mathrm{pc}^{-2}$ ), and the stellar mass surface density, $\mu_{\star}$ (in $M_{\odot} \mathrm{pc}^{-2}$ ).

Surface densities $\mu_{\star}$ and mass fractions $m_{j}$ reported in this paper are not corrected for the mass lost by stars during their evolution. This is justified because we also analyze SFRs, which count all mass turned into stars, even the mass that is eventually returned to the interstellar medium. The $M_{\star}$ values, however, do correct for this effect. The relation between the total mass turned into stars, $M_{\star}^{\prime}$, and the current mass in stars is $M_{\star}^{\prime} \sim 1.4 M_{\star}$ for the IMF used in this work.

Star formation histories can be expressed as the age distribution in either $\mathcal{L}_{\lambda 5635}$ or $\mu_{\star}$, obtained with the corresponding $\boldsymbol{x}$ and $\boldsymbol{m}$ arrays. These light and mass fractions themselves are representations of the SFH that is routinely used in fossil record studies (e.g., Tojeiro et al. 2007, 2009; Koleva et al. 2009). Star formation rates can also be defined. We worked with SFRs in both absolute $\left(M_{\odot} \mathrm{yr}^{-1}\right)$ and specific $\left(\mathrm{yr}^{-1}\right)$ units, as well as with surface densities, $\Sigma_{\mathrm{SFR}}$ (in $M_{\odot} \mathrm{yr}^{-1} \mathrm{pc}^{-2}$, sometimes called star formation intensities; e.g., Lehnert et al. 2015). These different ways of representing SFHs are complementary to each other, in the sense that they are useful to highlight different aspects of the results.

In addition to the organization of the STARLIGHT output in a format suitable for 2D work, PyCASso performs a number of other tasks that are useful in our analysis. In particular, radial distances $(R)$ to the nucleus are defined along elliptical rings, as explained in Cid Fernandes et al. (2013). As in previous papers, these distances are expressed in units of the half-light radius (HLR), defined as the semi-major axis length of the elliptical aperture that contains half of the total light of the galaxy at the rest-frame wavelength $5635 \AA$.

Finally, the galaxy masses $\left(M_{\star}\right)$ used in our analysis were obtained by adding the masses of each spatial zone, thus taking into account spatial variations on stellar populations and extinction. Masked spaxels that are due to foreground stars or other artifacts are corrected for in PyCASSO using the radial profile of $\mu_{\star}$, as explained in González Delgado et al. (2014b).

\subsection{Base of stellar population models}

This study introduces some novelties related to the base used in STARLIGHT to perform the spectral decomposition. Whereas previous works used a base of simple stellar populations (SSP, also known as instantaneous bursts), here we build a base of composite stellar populations (CSP) consisting of "square bursts", that is, episodes of constant SFR during a certain period of time and zero elsewhere. The SFR in each base component is scaled to form $1 M_{\odot}$ over the corresponding age interval.

We defined 18 such CSPs, spanning ages between 1 Myr and $14 \mathrm{Gyr}^{1}$, centered at $t_{j}=0.00245,0.00575,0.011,0.018,0.028$, $0.045,0.072,0.114,0.180,0.285,0.455,0.725,1.14,1.8,2.85$,

1 In the models of Vazdekis et al. (2015), the time interval between SSPs older than $10 \mathrm{Gyr}$ is $0.5 \mathrm{Gyr}$. To include in the CSP contributions from $13.7 \mathrm{Gyr}$, the age of the Universe, we include SSPs up to $14 \mathrm{Gyr}$ to generate the CSP with average age of $11.50 \mathrm{Gyr}$. 
R. M. González Delgado et al.: 2D star formation history of CALIFA galaxies

$4.55,7.25$, and 11.50 Gyr. These ages are separated by $\Delta \log t=$ 0.2 dex, except for the first two, which span 0.4 dex. A corollary of this logarithmic sampling is that $\Delta t$ grows with $t$. The last age bin, for instance, spans a $\Delta t=5.2$ Gyr long interval from 8.9 to 14.1 Gyr, while the penultimate bin spans $\Delta t=3.3 \mathrm{Gyr}$ (from 5.6 to $8.9 \mathrm{Gyr}$ ), and the bin before this covers the $\Delta t=2.1 \mathrm{Gyr}$ from 3.5 to $5.6 \mathrm{Gyr}$. On average, $\Delta t / t=0.46$, a property which must be kept in mind when evaluating the results of the synthesis.

An advantage of using CSPs instead of SSPs is that it makes the spectral fits less dependent on the specific SSPs that form the base. This is because each CSP includes all SSPs available in the given time interval, averaging the contribution of each SSP in proportion to its mass-luminosity ratio and the time intervals between the SSPs. In this way, the interpretation of the results in terms of SFR is more straightforward since for each time interval the SFR is constant on average. Another important advantage of this method is that the computation time to fit each spectrum is reduced by a factor 3-4 with respect to using SSP templates. The reason is that now we have only $18 \mathrm{CSPs}$ covering from $1 \mathrm{Myr}$ to 14 Gyr for each metallicity, instead of $\sim 35$ SSPs for the same age period, and the computational time required for STARLIGHT behaves as $N^{2}$, where $N$ is the number of components used for the spectral fit.

The CSP spectra were built using the set of SSP models from Vazdekis et al. (2015) for populations older than $t=63 \mathrm{Myr}^{2}$, and from the GRANADA models of González Delgado et al. (2005) for younger ages. The evolutionary tracks in the GRANADA models are those of Girardi et al. (2000), except for the youngest ages ( 1 and $3 \mathrm{Myr}$ ), which are based on the Geneva tracks (Schaller et al. 1992; Schaerer et al. 1993; Charbonnel et al. 1993); while the Vazdekis models are based on the BaSTI isochrones (Pietrinferni et al. 2004, 2006, 2009, 2013; Cordier et al. 2007). From these new sets of SSPs, we here used the base models that match the Galactic abundance pattern imprinted in the MILES stars (Sánchez-Blázquez et al. 2006). Eight metallicities were considered: $\log Z / Z_{\odot}=-2.28,-1.79$, $-1.26,-0.66,-0.35,-0.06,0.25$, and +0.40 . Each of the CSPs has the same initial chemical composition, and they are always affected by the same amount of extinction.

As in previous papers of this series, the initial mass function (IMF) is that of Salpeter, and dust is modeled as a foreground screen with a Cardelli et al. (1989) reddening law with $R_{V}=3.1$. We emphasize that the novelties reported above are essentially routine updates of the ingredients used in our full spectral synthesis with STARLIGHT. The results reported in this paper would remain essentially the same if they were computed with the bases used in our studies (e.g., González Delgado et al. 2016). The Appendix includes a comparison of the stellar population properties derived using CSPs or SSPs.

\subsubsection{Note on ages, lookback times, and redshifts}

The information on galaxy evolution analyzed here comes exclusively from the distribution of stellar population ages derived from our spectral fits. Because our galaxies are all basically at $z=0$, lookback times or the corresponding redshifts are equivalent to stellar ages, and are indeed treated as such

Owing to limitations in the number of $\mathrm{O}$ and $\mathrm{B}$ stars in the MILES library (Sánchez-Blázquez et al. 2006), the models of Vazdekis et al (2015) are uncertain for $t \leq 63 \mathrm{Myr}$, and in particular for metallicities below half solar; furthermore, BaSTI isochrones are not computed for ages younger than $10 \mathrm{Myr}$. In contrast, the models of González Delgado et al. (2005) are well optimized for young ages.
Table 1. Number of galaxies in each Hubble type and mass interval.

\begin{tabular}{lccccccc}
\hline \hline $\log M_{\star}\left(M_{\odot}\right)$ & $\mathrm{E}$ & $\mathrm{S} 0$ & $\mathrm{Sa}$ & $\mathrm{Sb}$ & $\mathrm{Sbc}$ & $\mathrm{Sc}$ & $\mathrm{Sd}$ \\
\hline $11.3-11.9$ & 29 & 11 & 9 & 6 & 1 & 1 & - \\
$11.0-11.3$ & 16 & 19 & 31 & 26 & 16 & 3 & 1 \\
$10.6-11.0$ & 8 & 15 & 12 & 26 & 33 & 6 & - \\
$9.8-10.6$ & 8 & 8 & 12 & 14 & 23 & 42 & 9 \\
$8.6-9.8$ & - & 1 & - & 1 & 1 & 21 & 26 \\
\hline $\operatorname{total}(436)$ & 61 & 54 & 64 & 73 & 74 & 73 & 36 \\
\hline$\left\langle\log M_{\star}\right\rangle$ & 11.2 & 11.0 & 11.0 & 10.9 & 10.7 & 10.1 & 9.5 \\
$\sigma\left(\log M_{\star}\right)$ & 0.4 & 0.4 & 0.4 & 0.4 & 0.4 & 0.5 & 0.5 \\
\hline
\end{tabular}

throughout this study. We say, for instance, that a stellar age of 4 Gyr corresponds to 4 Gyr in lookback time or $z \sim 0.4$.

Subtleties appear, however, when interpreting SFHs. A feature at a certain $t$, for example, an increase in the SFR at $t=$ $4 \mathrm{Gyr}$, does not necessarily imply that something happened in the galaxy itself $4 \mathrm{Gyr}$ ago. For all we know, this population could have formed elsewhere (e.g., a satellite) and accreted at any time between now and 4 Gyr ago. One expects the cosmic date $t_{e}$ of the event to leave a signature at $t=t_{e}$ in our SFHs only in events producing in situ star formation (like wet mergers). Dry mergers, on the other hand, will change the SFH at the typical age of the accreted stars.

Given that major and minor, dry and wet mergers are such conspicuous elements of the current paradigm for how galaxies assemble their stars over time, it is important to make this distinction.

\subsection{Case for a combined mass-morphology analysis}

Throughout the remaining paper, the SFHs derived with the method outlined above are studied as a function of stellar mass and Hubble type. The five bins in $M_{\star}$ and seven morphological bins defined for this purpose are given in Table 1, which lists the number of galaxies in this mass-morphology space. This approach can be justified in two different ways.

First, although morphology and $M_{\star}$ are related, the relation is not even approximately univocal. This is clearly seen in both Table 1 and in the upper panel of Fig. 2, where the distribution of $M_{\star}$ with morphology is shown. As for the general galaxy population, stellar masses decrease from (on average) $\log M_{\star}=11.2$ for ellipticals to 9.5 for the latest types, but the scatter is large. The dispersion within any Hubble type is $0.4-0.5 \mathrm{dex}$, as marked by the shaded band in Fig. 2. Conversely, the dispersion is also large in morphology for a fixed mass. In particular, the bins covering $\log M_{\star}$ between 9.8 and 11.3 are populated by all Hubble types from $\mathrm{E}$ to Sc. Hence, despite the evident correlation between $M_{\star}$ and morphology, these two properties cannot be considered approximately equivalent, which justifies our approach of investigating the effects of both of them upon our results.

A second motivation for studying SFHs as a function of both mass and morphology is illustrated in the bottom panel of Fig. 2, where we plot the mean age vs. mass diagram for our 436 galaxies, color-coded by morphology. In this plot the mean age of stars in a galaxy is represented by the mass-weighted mean log age, $\langle\text { log age }\rangle_{M}$, measured at $R=1 \mathrm{HLR}$ from the center. As shown by González Delgado et al. (2014b), properties at 1 HLR very closely match those defined for entire galaxies, so that the plot would hardly change if a galaxy-wide measure of $\langle\log \text { age }\rangle_{M}$ were used instead. We also note that this plot is the physical equivalent of the widely used color-magnitude diagram, where 

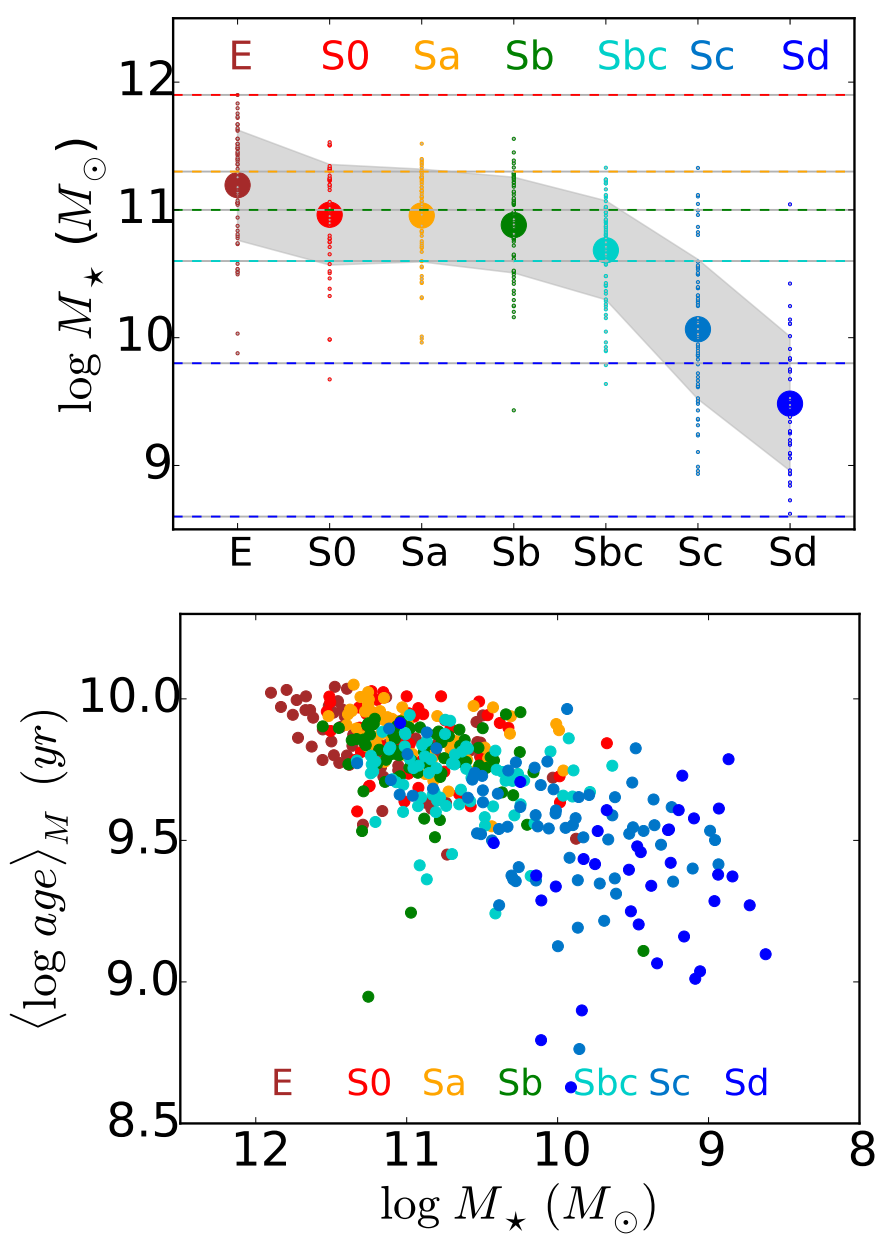

Fig. 2. Top: distribution of stellar mass with Hubble type. Large circles are the mean $M_{\star}$ for each Hubble type, and the gray area marks the onesigma dispersion in mass for each morphology. Horizontal lines mark the ranges of the five $M_{\star}$ bins defined in Table 1 that are used throughout the paper. Bottom: mass-weighted mean log stellar age versus $M_{\star}$ for the 436 galaxies in the sample, color-coded by the morphological type.

absolute magnitude and color play the roles of proxies for $M_{\star}$ and mean age, respectively.

Figure 2 shows that the mean age increases with mass, reflecting the well-documented pattern of galaxy "downsizing" (e.g., Gallazzi et al. 2005). The plot also shows that the spread in ages at any given $M_{\star}$ correlates with morphology, with earlier types tending to be older than later types of the same mass (see also González Delgado et al. 2015). Since the mean age of the stellar population is the first moment of a galaxy's SFH, this illustrates and justifies why our analysis needs to be conducted in terms of both $M_{\star}$ and Hubble type.

\section{Spatially resolved star formation histories}

This section presents our results for the spatially resolved SFH as a function of galaxy mass and morphological type. This involves mapping some SFH-function (such as luminosity, mass, or SFR) in terms of two spatial coordinates and lookback time $t$, and its dependence on two further variables, $M_{\star}$ and Hubble type. Even after collapsing the 2D $(x, y)$ information into a single spatial dimension $R$, we are left with a complex manifold whose exploration poses challenges to visualization and scientific analysis.

We chose to first present our results in their most complete form by means of the $R \times t$ diagrams first introduced by Cid Fernandes et al. (2013) as a means of compressing the spatially resolved SFH of galaxies in images (Sect. 4.1). These diagrams contain essentially all the science discussed in this paper, but fully comprehending them requires summarizing the information in simpler forms. One way of carrying out such a simplification is by discretizing the radial and temporal dimensions into a few bins, and this is also presented here (Sect. 4.2). Projections of the $\operatorname{SFH}\left(t, R, M_{\star}\right.$, morphology) manifold that are easier to grasp are presented in Sect. 5.

\section{1. $R \times t$ maps}

For a generic SFH-related function $F(x, y, t, Z)$, with age, metallicity, and spatial dimensions, the $R \times t$ map is obtained by first collapsing $F$ along the $Z$ axis, and then azimuthally averaging the $F(x, y, t)$ image along elliptical annulli of varying $R$. The result is a map in $(R, t)$ space. Cuts at fixed $t$ give radial profiles of $F$ at the given lookback time, while for a constant $R$ we obtain the distribution of $F$ among the populations of different ages. For visualization purposes, and also to mitigate degeneracies intrinsic to stellar population synthesis (e.g,. Worthey 1994; Cid Fernandes et al. 2005; Asari et al. 2007), we applied a Gaussian smoothing of 0.5 dex in FWHM in $\log t$. As in our previous studies (e.g., Pérez et al. 2013; Cid Fernandes et al. 2013; González Delgado et al. 2014b), $R$ is expressed in units of the galaxy HLR, a convenient metric when averaging radial information for different galaxies.

Figure 3 presents two sets of $R \times t$ maps that represent the mean SFH obtained for galaxies in bins in the mass-morphology space. The Hubble type runs from $\mathrm{E}$ to $\mathrm{Sd}$ from left to right, while $M_{\star}$ increases upward. The layout is exactly the same as that of Table 1, except that bins containing fewer than 8 galaxies are not shown. Diagrams in the top half of Fig. 3 express the 2D $\mathrm{SFH}$ in terms of the continuum luminosity per unit area $\left(\mathcal{L}_{\lambda 5635}\right)$ associated with stars of a given age and radial position, as indicated in the reference panel in the top right corner, where the average for all 436 galaxies is shown. Diagrams in the bottom half of the figure show the corresponding images in terms of stellar mass surface density $\left(\mu_{\star}\right)$.

Some clear differences in the SFHs along the Hubble sequence and galaxy mass are visible in these plots. For instance, for a fixed Hubble type, we see progressively more extended SFHs toward lower $M_{\star}$; this is the signature of downsizing. For galaxies in a same $M_{\star}$ bin, SFHs appear to be more extended in time among later Hubble types. This effect is more clearly visible in the outer regions, which are associated with the disks of spirals, than at small $R$, where the regions are presumably dominated by the bulges or the thick disks.

The richness of information in these maps is huge. It can be somewhat overwhelming, in the sense that it can be hard to interpret and is strongly dependent upon seemingly unimportant choices, like whether to plot absolute or specific quantities, to show light- or mass-related properties, and use logarithmic or linear scales. The next sections therefore present basically the same information as is contained in Fig. 3, but expressed in ways that allow a fuller understanding of the results. 
R. M. González Delgado et al.: 2D star formation history of CALIFA galaxies
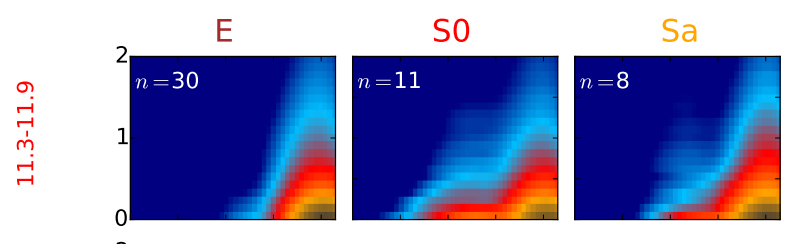

$\mathrm{Sb}$

$\mathrm{Sbc}$

Sc

Sd
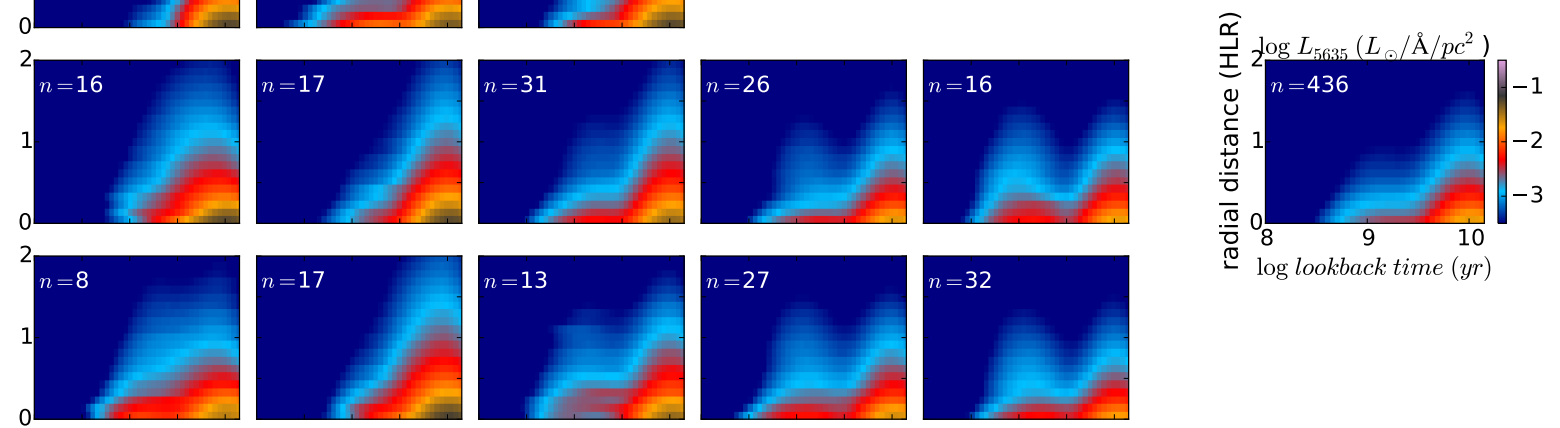

- $\log$ lookback time $(y r)$

$$
\text { 卢 }
$$
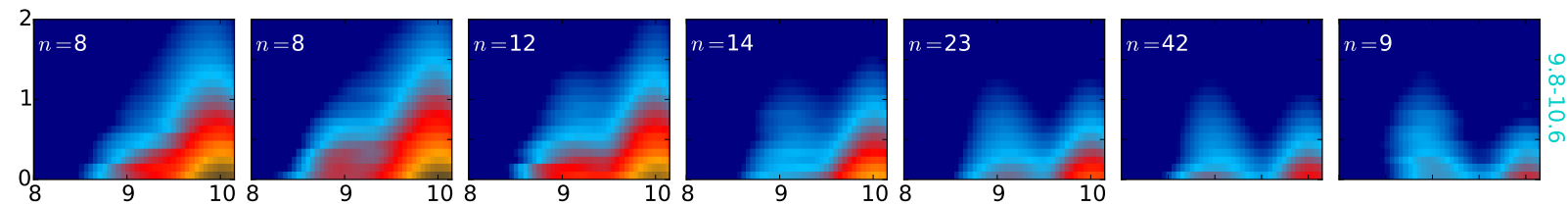

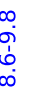

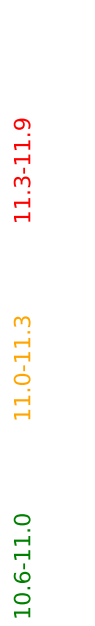

E
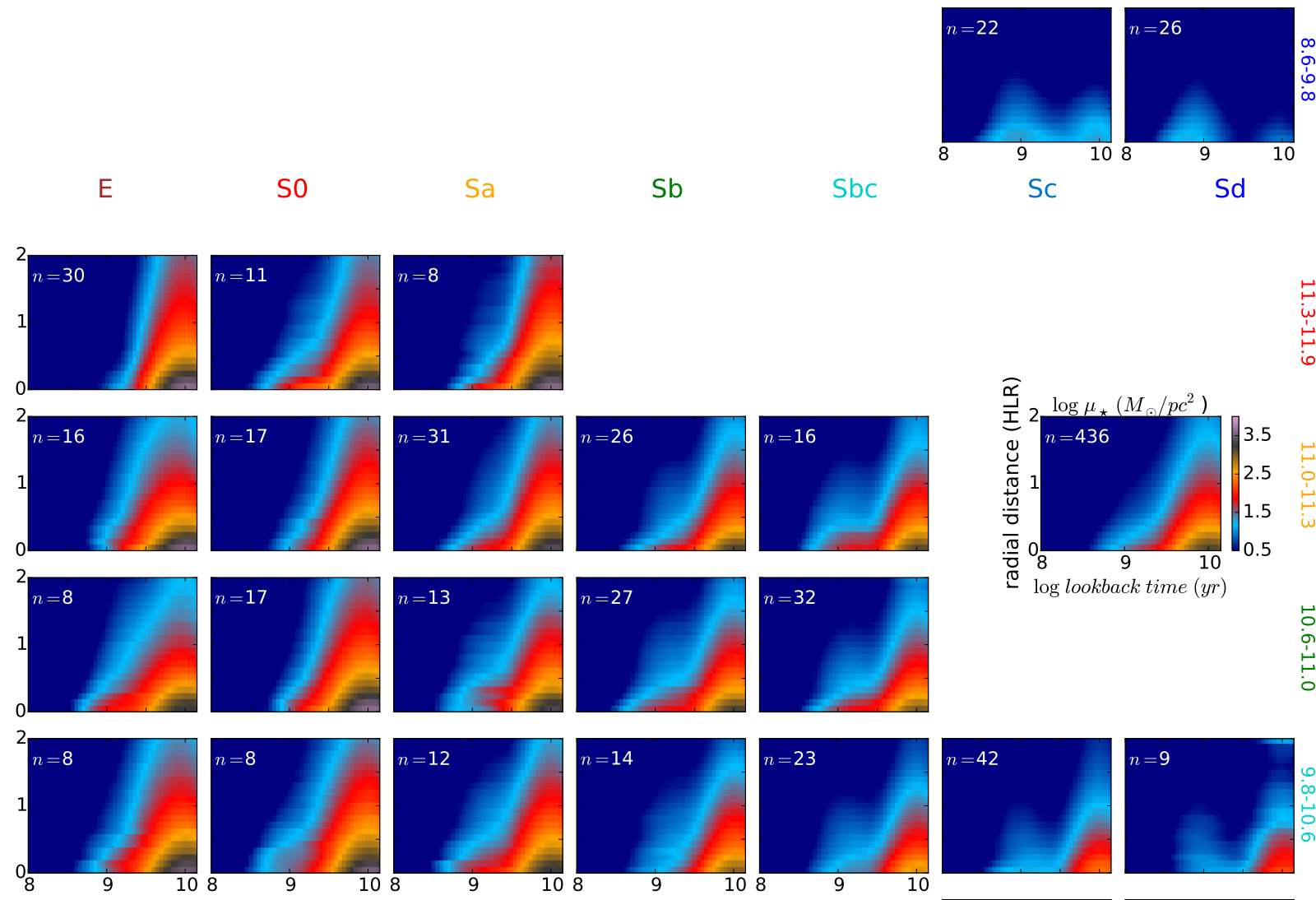

$\mathrm{Sbc}$
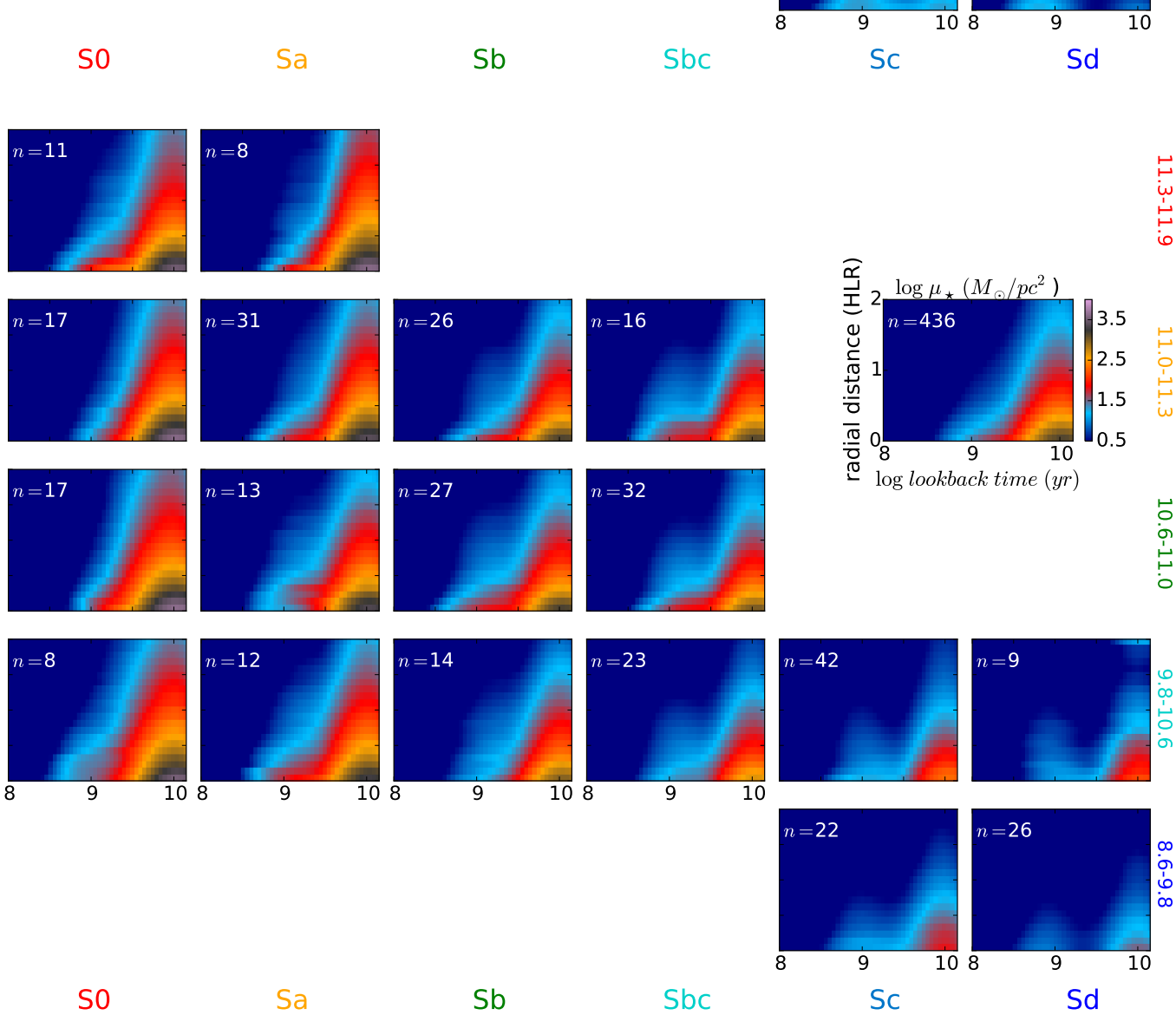

$\mathrm{Sb}$

Fig. 3. $R \times t$ diagrams showing azimuthally averaged SFHs in terms of distributions of light and mass as a function of the stellar ages (= lookback time) and distance to the nucleus (in HLR units). The intensity of the map shows the luminosity (corrected for extinction) at $5635 \AA$ per unit area $\left(\mathcal{L}_{\lambda 5635}\right.$, upper panels $)$, and stellar mass formed per unit area $\left(\mu_{\star}\right.$, bottom panels $)$. Each frame represents the average results for bins in the $M_{\star}$-morphology plane, with stellar masses decreasing from the top down, and Hubble type running from $\mathrm{E}$ to Sd from left to right. The number of galaxies is indicated in each panel. Average maps for the entire sample are shown in the top-right panels, which also show the color bar. 


\subsection{Stellar population components}

The light $(x)$ and mass $(m)$ fractions are two further examples of $F(x, y, t, Z)$ functions that can be represented in $R \times t$ diagrams like those in Fig. 3. Instead, Fig. 4 presents an alternative way of visualizing results for these two functions by discretizing both the $R$ and $t$ axes into a few relevant ranges. Each panel shows three rectangular "bar charts", each corresponding to a spatial region: $R<0.5 \mathrm{HLR}$ (left), $0 \leq R<2$ HLR (middle), and $R>1.5$ HLR (right). The middle charts are meant to represent the galaxy as a whole, while those on the right represent distances to the nucleus similar to that of the solar neighborhood, since $1.75 \mathrm{HLR}$ is $\sim 8 \mathrm{kpc}$ for the mean HLR of the CALIFA SbcSc galaxies. The age information is compressed into four colorcoded representative ranges: $t \leq 1 \mathrm{Gyr}$ (blue), $1<t \leq 4 \mathrm{Gyr}$ (green), $4<t \leq 9 \mathrm{Gyr}$ (orange), and $t>9 \mathrm{Gyr}$ (red). In terms of redshift, these ranges correspond to approximately $z \leq 0.1$, $0.1<z \leq 0.4,0.4<z \leq 1.5$, and $z>1.5$, respectively. For reference, these cosmic epochs can be associated with (i) the present time; (ii) the growth of the thin disk in spirals; (iii) the size growth epoch; and (iv) the early formation of the galaxy halo and/or core.

The layout of Fig. 4 is again the same as in Table 1, with Hubble type varying from column to column and $M_{\star}$ decreasing from the top to bottom rows. Panels in the top half show the percentage contributions in light of the populations in these four age ranges and three spatial regions. These are discussed first.

\subsubsection{Light fractions}

The top panels of Fig. 4 show a steady progression of young populations (blue, $t \leq 1 \mathrm{Gyr}$ ) along the Hubble sequence, a variation that is stronger than with stellar mass. Breaking down the evolutionary information in terms of radial locations, we find the following.

1. Galaxy-wide average ( $R<2$ HLR, middle charts). The contribution of $<1 \mathrm{Gyr}$ populations varies from $\sim 1 \%$ in $\mathrm{E}$ to $\sim 60 \%$ in Sd systems, while it hardly changes (23 to $21 \%$ ) from the least to the most massive $\mathrm{Sb}$. Old populations show the opposite behavior, with contributions of $>4$ Gyr stars (orange and red) increasing from $20 \%$ for $\mathrm{Sd}$ to $90 \%$ for the most massive E. These populations show a stronger dependence on $M_{\star}$ in Sa galaxies, increasing from 58 to $80 \%$ over the $M_{\star}$ range we sampled, and similarly for ellipticals. S0 galaxies, however, do not show any systematic dependence on $M_{\star}$, as is also shown in Fig. 3.

2. Outer regions $(1.5<R<2$ HLR, right charts). $x(t<1$ Gyr $)$ ranges from $\sim 3 \%$ in $\mathrm{E}$ to $\sim 65 \%$ in $\mathrm{Sd}$, but only from 29 to $24 \%$ from the least to the most massive Sb. Stars older than $4 \mathrm{Gyr}$ increase their contribution from $20 \%$ for Sd to $86 \%$ for the most massive $\mathrm{E}$. These results are very similar to those for the $R=0-2$ HLR region, indicating that in terms of light, the "disk" plays a dominant role in SFHs that are derived for entire galaxies.

3. Central region ( $R<0.5$ HLR, left charts). In this region, $x(t<1 \mathrm{Gyr})$ is smaller and $x(t>4 \mathrm{Gyr})$ is larger than in the "disk regions" for all morphological types and $M_{\star}$ bins. This is also the case for the mass fractions (lower panels). This is a clear indication of the inside-out growth process in CALIFA galaxies (Pérez et al. 2013; González Delgado et al. 2015), although this is not so evident in the two extreme morphology- $M_{\star}$ bins, namely, Sd galaxies with $\log M_{\star}=$ 8.6-9.8 and $\mathrm{E}$ galaxies with $\log M_{\star}=11.3-11.9$. In the younger populations, $x(t<1 \mathrm{Gyr})$ grows from $\sim 1 \%$ in
E galaxies to $13 \%$ in Sb galaxies and $57 \%$ among Sd galaxies. Stars older than $4 \mathrm{Gyr}$ vary from $23 \%$ in Sd galaxies to $96 \%$ in massive ellipticals. The dependence on $M_{\star}$ is very clear for ETGs, for which $x(t>4 \mathrm{Gyr})$ changes from $64 \%$ to $91 \%$ from the least to the most massive Sa galaxies, and similarly for E galaxies.

\subsubsection{Mass fractions}

Panels in the bottom half of Fig. 4 show that most of the stellar mass formed very early on, with very little mass in stars younger than $1 \mathrm{Gyr}$. Sd and the less massive Sc galaxies are exceptions, with $m(t<1 \mathrm{Gyr})$ fractions of up to $20-30 \%$, with a tendency to increase toward the outer regions. The main tendencies seen in these plots are summarized below.

1. Galaxy-wide average $(R<2$ HLR). Except for the latest types, the fractions in the $>9$ Gyr bin (painted in red) are the largest amongst our four age-ranges, with $m(t>9 \mathrm{Gyr}) \sim$ 40-70\%. This old component increases with $M_{\star}$. The contribution of 4-9 Gyr stars (orange) shows a clear tendency to increase from late to early Hubble types, with little or no dependence on $M_{\star}$. Stars in the 1-4 Gyr range (green) generally account for $\$ 20 \%$ of the mass, with a tendency to decrease in strength at the highest $M_{\star}$ bins.

2. Outer regions $(1.5<R<2$ HLR). The mass fractions in these outer regions behave similarly to the previous case (0-2 HLR). The oldest populations decrease their fractions slightly (signaling negative mean age gradients that were explicitly studied in González Delgado et al. 2015), but still account for much of the mass. Noticeably, the $m(t>9 \mathrm{Gyr})$ fractions for E and S0 are smaller than for Sa, while their 4-9 Gyr populations are larger.

3. Central region $(R<0.5 \mathrm{HLR}) . t>9$ Gyr stars dominate the mass in the central regions, accounting for over $60 \%$ in most spirals and up to $80 \%$ in the most massive galaxies. The exceptions are, again, Sd and low-mass Sc galaxies. Except at the highest $M_{\star}$ bin, this old component contributes less in E and S0 galaxies

\section{SFH as a function of mass and Hubble type}

After presenting the variation of our 2D SFHs with galaxy mass and Hubble type, we now simplify the analysis by investigating variations as a function of $M_{\star}$ or morphology separately. These projected views of SFHs in the mass-morphology space aid in the interpretation of the results presented above.

This section presents SFHs in terms of mass fractions (Sect. 5.1), absolute (Sect. 5.2) and specific (Sect. 5.3) SFRs, and star formation intensities (Sect. 5.4) as a function of lookback time. In order to emphasize evolutionary aspects, the spatial analysis is simplified to the same three radial regions as were used in the discussion of Fig. 4, namely, $R<0.5$ HLR, 0-2 HLR, and 1.5-2 HLR, corresponding to the nuclear regions, the whole galaxy, and the outer regions.

\subsection{SFHs: Mass fractions}

Figure 5 shows stellar mass fractions $(m)$ as a function of lookback time. The left, middle, and right panels correspond to the whole galaxy and the central and outer regions, respectively. The top panels show the average $m(t)$ curves for galaxies in our five $M_{\star}$ bins, while the panels in the bottom stack show objects by 
R. M. González Delgado et al.: 2D star formation history of CALIFA galaxies
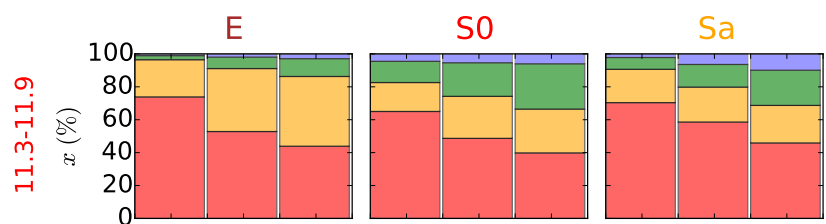

$\mathrm{Sb}$

$\mathrm{Sbc}$
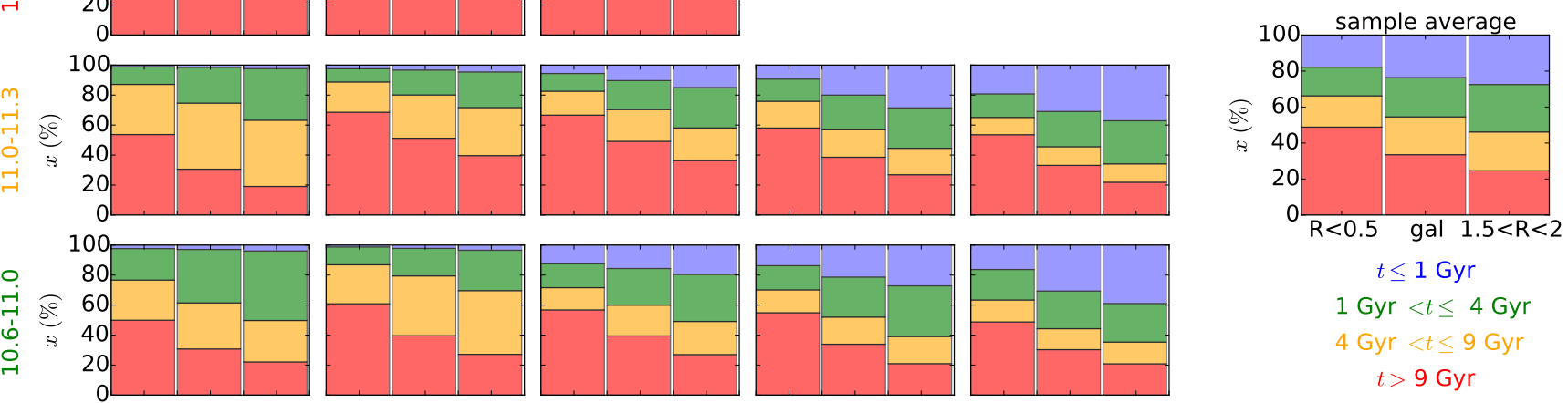

$$
t \leq 1 \mathrm{Gyr}
$$

$1 \mathrm{Gyr}<t \leq 4 \mathrm{Gyr}$

$4 \mathrm{Gyr}<t \leq 9 \mathrm{Gyr}$ $t>9 \mathrm{Gyr}$
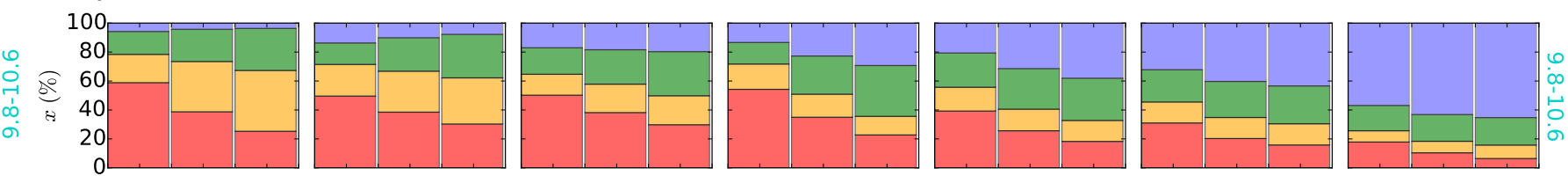

$\infty$
$\stackrel{9}{0}$
$\dot{0}$
$\infty$

E
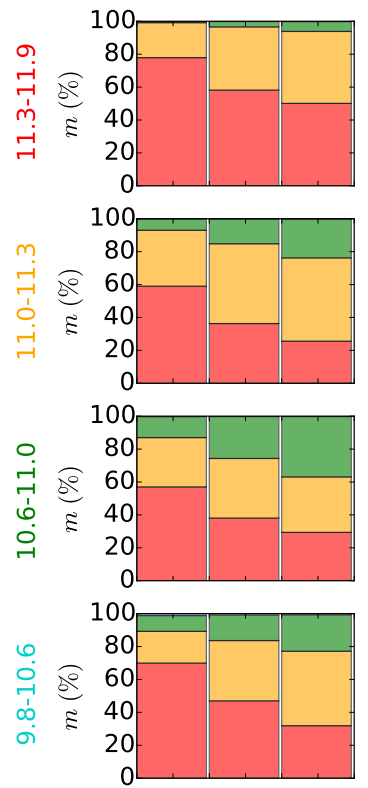
$\infty$
0
0
0
$\infty$ so
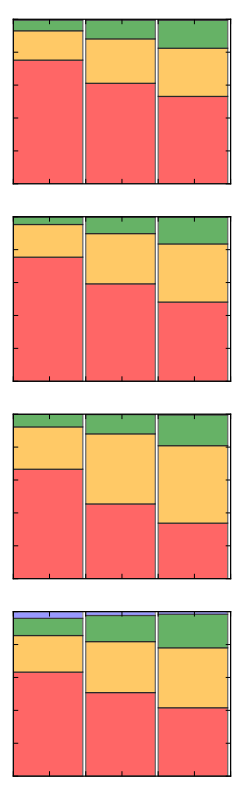
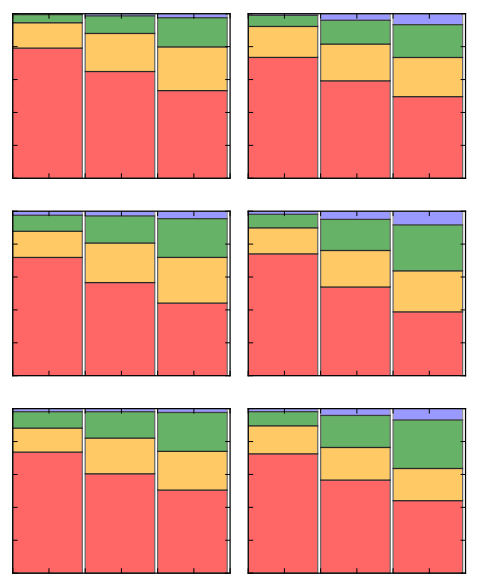

$\mathrm{Sb}$

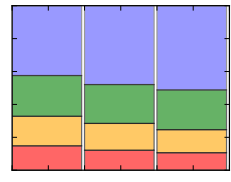

Sc

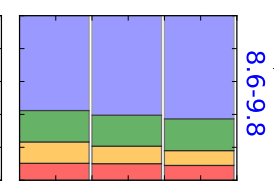

Sbc
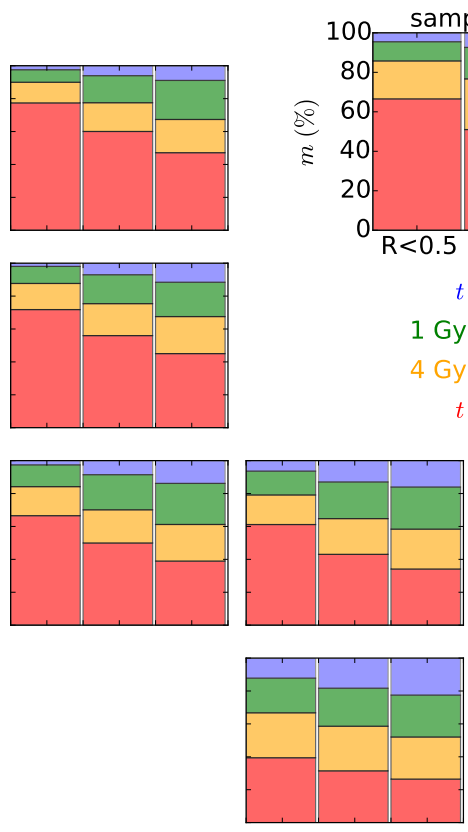

Sc

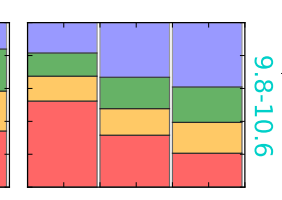

Fig. 4. Average light (upper panels) and mass (bottom) fractions due to stars in different age and radial ranges. Each panel corresponds to a bin in the galaxy mass-morphology plane, exactly as in Fig. 3. The three-bar chart histograms in each panel correspond to different galaxy regions: the inner region $R \leq 0.5 \mathrm{HLR}$ (left bar); the whole galaxy $R \leq 2 \mathrm{HLR}$ (central), and outer regions $1.5<R<2$ HLR (right). Colors represent the following age ranges (in Gyr): $<1$ in blue, 1-4 in green, 4-9 in orange, and $>9$ in red. Panels in the top right corner show averages for the full sample. 

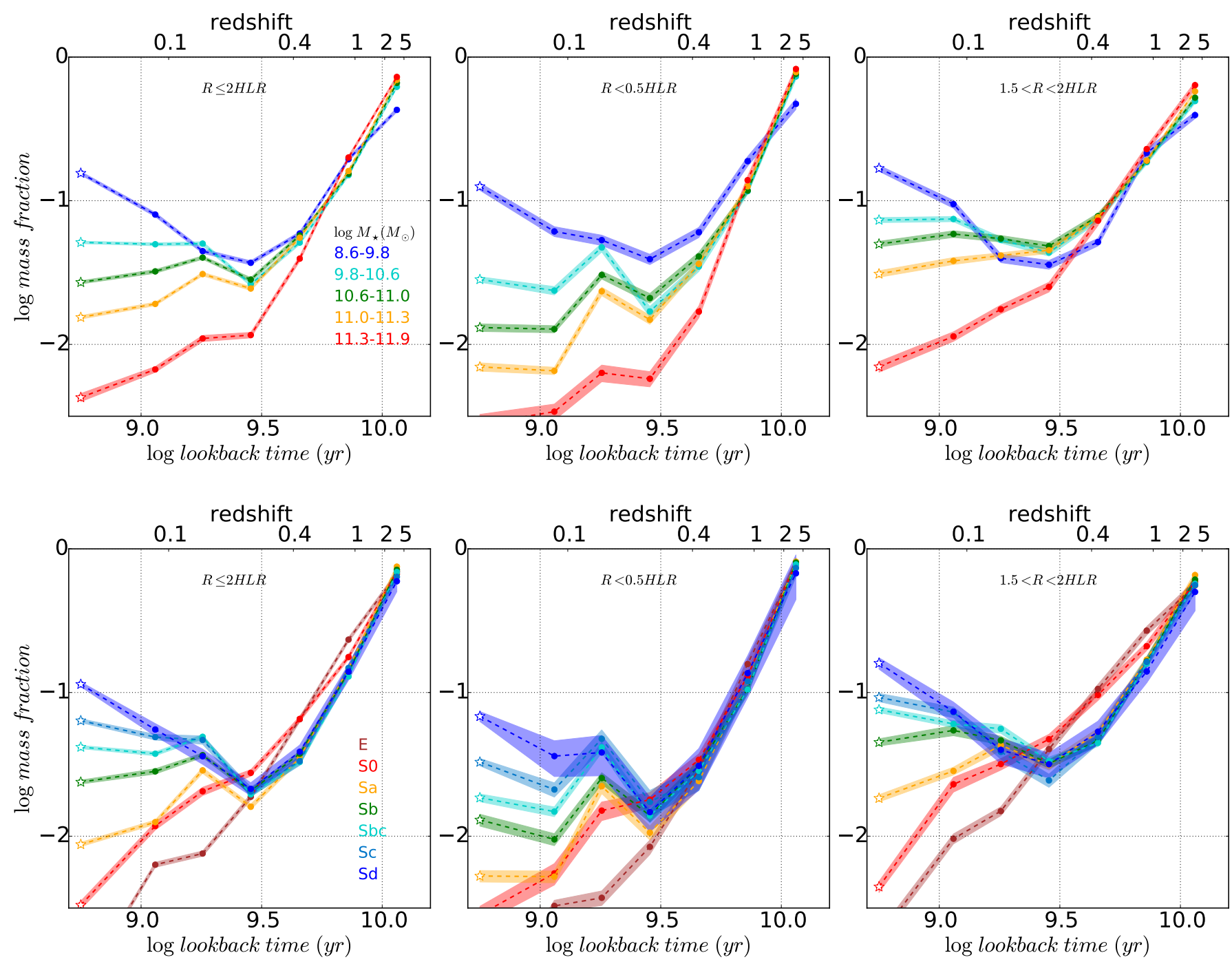

Fig. 5. The SFH is represented here by the fraction of stellar mass formed in each epoch, $m(t)$, averaged over different $M_{\star}$ (upper panels) and Hubble type (bottom) bins. Left, middle, and right panels show the average $m(t)$ curves for different regions in the galaxy: the whole galaxy (left), the inner regions (middle), and the outer regions (right). The shaded bands around the mean curves represent \pm the error in the mean, computed as the rms dispersion of the corresponding $m(t)$ values divided by the square root of the number of galaxies in each bin.

Hubble type. These curves are histograms, with each point representing a 0.2 dex wide bin in $\log t$, but it is visually clearer to connect the points. We also note that because of the logarithmic sampling in $t$ and because these are not masses but mass fractions, the results in Fig. 5 should not be read as $\operatorname{SFR}(t)$ curves. SFRs are presented in the next section.

Inspection of the top panels show that the highest mass fractions invariably occur at the earliest times. Subsequent star formation varies systematically with $M_{\star}$, with the low $M_{\star}$ galaxies forming stars over extended periods of time, and high $M_{\star}$ galaxies exhibiting the fastest decline in $m(t)$. This footprint of cosmic downsizing is more clearly observed in the inner regions (central panels), which (except for the latest Hubble types) are mainly associated with the spheroidal component. The decline in $m(t)$ of the most massive galaxies is visibly faster in the inner regions than away from the nucleus. The curves for the outer regions are similar to those obtained for the galaxy as a whole.

The bottom panels of Fig. 5 show that the behavior with morphology mimics the behavior with $M_{\star}$, with $m(t)$ peaking at the earliest time and subsequent star formation increasing systematically from early to late types. However, there are at least two important differences. (a) In the inner regions, the $m(t)$ curves are very similar from their peak at the oldest ages to $\sim 4 \mathrm{Gyr}$ ago. After this epoch, $m(t)$ curves have the same qualitative behavior, whether binned by $M_{\star}$ or morphology, with ellipticals and massive galaxies declining rapidly, while spirals and low $M_{\star}$ systems stretch their star formation activity over a longer period of time. (b) In the outer regions, E and S0 galaxies have higher mass fractions than later types in the $t=4$ and 7 Gyr age bins. Our analysis does not reveal whether these stars were formed in situ at these epochs or accreted from other systems, but it does show that they are now part of the envelopes of E and S0 galaxies.

In a first approximation, the decline of $m(t)$ in spirals and inner regions of $\mathrm{E}$ and $\mathrm{S} 0$ at early times can be fitted by a decaying function of cosmic time much like the so-called $\tau$-models used in parametric modeling of SFHs, particularly in high- $z$ studies (Maraston et al. 2010, 2013). However, the SFHs in Fig. 5 clearly show that additional star formation has taken place at $t<4$ Gyr. Evidence of such extended phases of star formation has been reported in the literature. For instance, a global star-forming episode in the last 2-4 Gyr has been detected in the disk of M31 (Williams et al. 2015; Bernard et al. 2015). Furthermore, from modeling the stellar populations of a sample of spiral galaxies with $M_{\star}>10^{10}$, Huang et al. (2013) found that in 

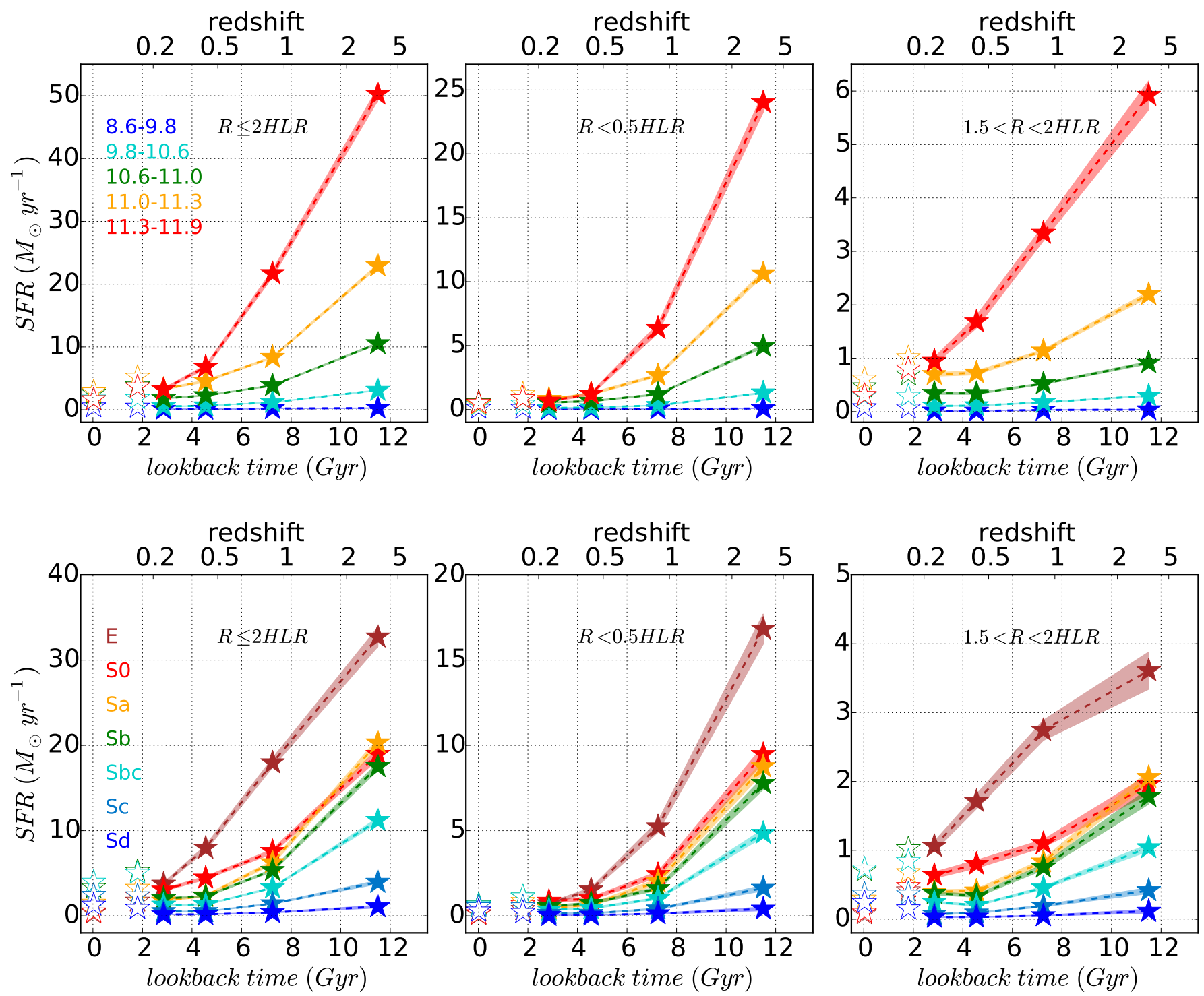

Fig. 6. The SFR in each epoch averaged for different bins in stellar mass (upper panels) and Hubble type (bottom). As in Fig. 5, the left, middle, and right panels show results for the whole galaxy, the inner $R<0.5 \mathrm{HLR}$, and radial distances between 1.5 and 2 HLR, respectively. Filled stars show SFR values at the original $t$-sampling of our base of composite stellar populations. The open star at $t=1.8$ Gyr shows the SFR obtained by averaging over all populations younger than 2.2 Gyr. Similarly, the open star at the left end of the SFR $(t)$ curves shows the value obtained by averaging over components younger than $32 \mathrm{Myr}$. The shaded regions represent the statistical uncertainty on the mean curves.

addition to an exponentially declining SFR, the observed $D_{4000}$ break and $\mathrm{H}_{\gamma}$ indices require an additional burst of star formation in the last 2 Gyr to be adequately fitted. This burst is more significant in the outer disk of their galaxies, and accounts for a small fraction of the total mass formed. Such late bursts of star formation are also found in other contexts, for instance, in the photometric analysis of $z<1.2$ galaxies by Walcher et al. (2008). Our results support these earlier findings, and show the advantages of obtaining the SFH with non-parametric methods.

\subsection{SFHs: Star formation rates}

The SFR is calculated at each epoch as the ratio of the stellar mass formed to the duration of the "square burst" event represented by the corresponding CSP in our base. These are therefore time-averaged SFRs during each time interval. Although mathematically related to the mass fractions discussed above, $\operatorname{SFR}(t)$ curves are perhaps easier to interpret, in addition to being more directly connected to observables. We recall that as previously noted in Sect. 3.2.1, we cannot know whether the stars within a given age bin $t$ were formed in situ at lookback time $t$ or were accreted from other systems at any time since $t$. This caveat should be kept in mind when evaluating our SFR $(t)$ curves and indeed all the SFH descriptors explored in this paper.

Figure 6 shows the time evolution of the SFR for $M_{\star}$ (top panels) and morphology bins (bottom), again divided into global (left), central (middle), and outer (right) regions. The plot shows that SFRs decline rapidly as the Universe evolves. The top panels show that at any epoch, the SFR scales with $M_{\star}$. The most massive galaxies thus have the highest SFRs, reaching $\sim 60 M_{\odot} \mathrm{yr}^{-1}$ at $z \gtrsim 2$, declining by a factor 2 at $z=1$, and by a factor $>10$ by the time we reach $z=0$. In low-mass galaxies the SFR at early epochs is quite low, $\sim 0.3 M_{\odot} \mathrm{yr}^{-1}$, rising by a factor of $\sim 2$ in recent times (hardly noticeable because of the scale).

The SFR in the inner regions (top central panels) shows an even faster decline than the total (whole-galaxy) SFR $(t)$. At early 
epochs, this inner region contributes significantly $(\sim 40 \%)$ to the total SFR, except for the lowest $M_{\star}$ galaxies, for which the $R<0.5$ HLR accounts for only a quarter of the total SFR. In contrast, $\operatorname{SFR}(R<0.5 \mathrm{HLR})$ currently represents $<20 \%$ of the total. SFRs also decline with time in the outer regions (right panels), although this decline is only significant for our highest $M_{\star}$ bin. In galaxies with $M_{\star}<10^{11}$, these outer regions can be approximated by a roughly constant SFR throughout the ages. We also note that at early times, these regions account for only $\sim 10 \%$ of the galaxy SFR.

Averaging the $\operatorname{SFR}(t)$ functions by morphology (bottom panels in Fig. 6) leads to similar results as averaging by $M_{\star}$, in particular for the inner regions. As a consequence of the relation between morphology and mass (Fig. 2), the scaling of SFRs with $M_{\star}$ is preserved with Hubble types, with ellipticals showing the highest SFRs at $z>0.5$. The S0, Sa, and Sb galaxies, which span similar $M_{\star}$ values (see Table 1), have almost identical SFRs at early times.

The SFR in the outer regions show interesting differences with respect to the inner regions and with respect to the stacking by $M_{\star}$. While in most cases, $\operatorname{SFR}(t)$ can be approximated as exponentially decaying for lookback times between 12 and $4 \mathrm{Gyr}$, the $\operatorname{SFR}(1.5<R<2 \mathrm{HLR})$ in ellipticals cannot be explained by these simple laws, as is shown by the excess SFR between $\sim 4$ and 7 Gyr ago.

This suggests that E galaxies are actively forming (or accreting) stars in their outskirts between $z=1.5$ and 0.5 . S0 galaxies also show an excess of SFR at these epochs with respect to galaxies of similar mass, like $\mathrm{Sa}$ and $\mathrm{Sb}$ galaxies. This same effect was noted in the bottom right panel of Fig. 5, where both E and S0 galaxies show larger mass fractions in the 4 and 7 Gyr bins.

Another interesting feature shown in Fig. 6 is the increase in $\operatorname{SFR}(1.5<R<2 \mathrm{HLR})$ in the last $3 \mathrm{Gyr}$ of spirals. This effect is more evident in Sb-Sc galaxies, and suggests that the disk of spirals has experienced a rejuvenation in the last $3 \mathrm{Gyr}$, achieving SFRs similar to those of earlier epochs. Overall, however, the outer disks of late-type spirals seem to have undergone relatively little variation in the levels of SFR throughout their lives.

\subsection{SFHs: Specific star formation rates}

Yet another way to express SFHs is through the time evolution of specific SFRs. For a galaxy today, the sSFR is usually defined as $S F R$ (today) $/ M_{\star}$. Because of the slightly sublinear relation between SFR and $M_{\star}$ (the MSSF; SFR $\left.\propto M_{\star}^{0.7-0.9}\right)$, the present-day sSFR declines slowly with mass, with star-forming galaxies occupying a tight sequence in the sSFR vs. $M_{\star}$ space. ETGs fall below this sequence and display a large spread of sSFRs at fixed $M_{\star}$ (Schiminovich et al. 2007; Salim et al. 2007; Karim et al. 2011). Spatially resolved data allow us to extend this concept and define a local sSFR, which is computed as the ratio between the surface densities of SFR and stellar mass, $s S F R=\Sigma_{\mathrm{SFR}} / \mu_{\star}$. González Delgado et al. (2016) used CALIFA data to show that this local sSFR increases with $R$, and that at the present time it grows faster with radius within $R<1$ HLR than outward, probably signaling the bulge-disk transition. Mapping how sSFRs vary as a function of radial location is therefore useful for galaxy evolution studies.

Our fossil record analysis allows us to proceed one step further and study temporal variations of the sSFR. We do this by computing $S S F R(t)=S F R(t) / M_{\star}^{\prime}(t)$, that is, the SFR at lookback time $t$ (discussed in the previous section) divided by the stellar mass formed up to that epoch. We note that we prefer to use $M_{\star}^{\prime}$, which includes all the stellar mass ever formed, instead of the mass still in stars $\left(M_{\star}\right)$ in the definition of the sSFR. Defined in this way, the inverse sSFR gives a doubling-mass time-scale, i.e., the time span required to form as much mass as a galaxy has acquired to date at the current rate. In addition, the product of the SSFR and the time since the onset of star formation $(T$, in practice the age of the Universe) gives the familiar birthrate parameter, $b=s S F R \times T$ (Scalo 1986; Cid Fernandes et al. 2013). In practice, $M_{\star}^{\prime} \sim 1.4 M_{\star}$ for a Salpeter IMF, so multiplication by 1.4 converts our values into the usual (but less natural) definition. The reason is that it takes only $\sim 4$ Gyr for a stellar population to lose $1 / 1.4$ of its original mass. Since most stars are older than this, a correction factor of 1.4 accurately converts original into current stellar masses.

The results are shown in Fig. 7, where, as in the two previous figures, the top and bottom panels average galaxies by current stellar mass and morphology, respectively, with galaxywide, central, and outer regions presented in the panels from left to right.

First of all, we clarify why, by construction, all curves start from the same point, $s S F R=0.19 \mathrm{Gyr}^{-1}$ at lookback time 11.5 Gyr. This occurs because the mass formed in this first bin appears both in the numerator and denominator, such that the SSFR value obtained is simply the inverse of the $\Delta t=5.2$ Gyr time-span of this first bin (see Sect. 3.2). This mathematical triviality exposes a well-known limitation of archeological studies. Because the spectral evolution of stellar populations approximately follows a logarithmic clock, codes like STARLIGHT are unable to distinguish populations of comparable ages (Ocvirk et al. 2006; Tojeiro et al. 2009). In particular, we cannot resolve SFHs to better than a few Gyr for ages $\gtrsim 4 \mathrm{Gyr}$. Our estimates of the sSFR at these long lookback times is therefore averaged for a period of time that is significantly longer than the time sampled by high- $z$ studies that are based on $\mathrm{H} \alpha$, UV, or FIR emission (which sample timescales of 0.1 Gyr or less), a caveat that must be taken into account when comparing results.

Figure 7 shows that sSFRs decrease as the Universe evolves. This decline has been observed in archeological studies similar to ours (e.g,. Asari et al. 2007; McDermid et al. 2015) and in many redshift surveys (Speagle et al. 2014). These cosmological studies found that the sSFR increases with redshift as $(1+z)^{3}$ for $z<2$ (Elbaz et al. 2007; Daddi et al. 2007; Oliver et al. 2010; Rodighiero et al. 2010; Elbaz et al. 2011, e.g). Our sSFRs decline more slowly with time, following roughly $(1+z)^{2}$ in the highest $M_{\star}$ bin, but this comparison is probably plagued by the resolution issues discussed above. For the same reason, we cannot possibly resolve the initial plateau or rising in SSFR between $z=2$ and 6 (Feulner et al. 2005; Magdis et al. 2010; Stark et al. 2013; Lehnert et al. 2015).

The current sSFRs in Fig. 7 were calculated considering all components younger than 2.2 Gyr in a single time-bin. This gives $s S F R \sim 0.07 \mathrm{Gyr}^{-1}$ for galaxies with $10^{10} \leq M_{\star} \leq$ $10^{11}$. In terms of the more conventional definition $\left(S F R / M_{\star}\right)$, this corresponds to $0.1 \mathrm{Gyr}^{-1}$, typical of galaxies in the MSSF (Brinchmann et al. 2004; Salim et al. 2007). These values are also in agreement with our estimates that are based on timescales of $32 \mathrm{Myr}$ (for which our SFRs match those obtained from $\mathrm{H} \alpha$; González Delgado et al. 2016). In terms of time evolution, we find that there is an increase in SSFR in last few Gyr in galaxies with $M_{\star}<10^{11}$. This rejuvenation is also observed in Fig. 5 .

In terms of the dependence on galaxy mass, the top left panel in Fig. 7 shows that galaxies on the whole follow quite similar $\operatorname{sSFR}(t)$ curves down to $\sim 3$ Gyr ago, where the curves split into the well-documented downsizing pattern, with SSFR and $M_{\star}$ parting ways. This small dependence on $M_{\star}$ indicates that 

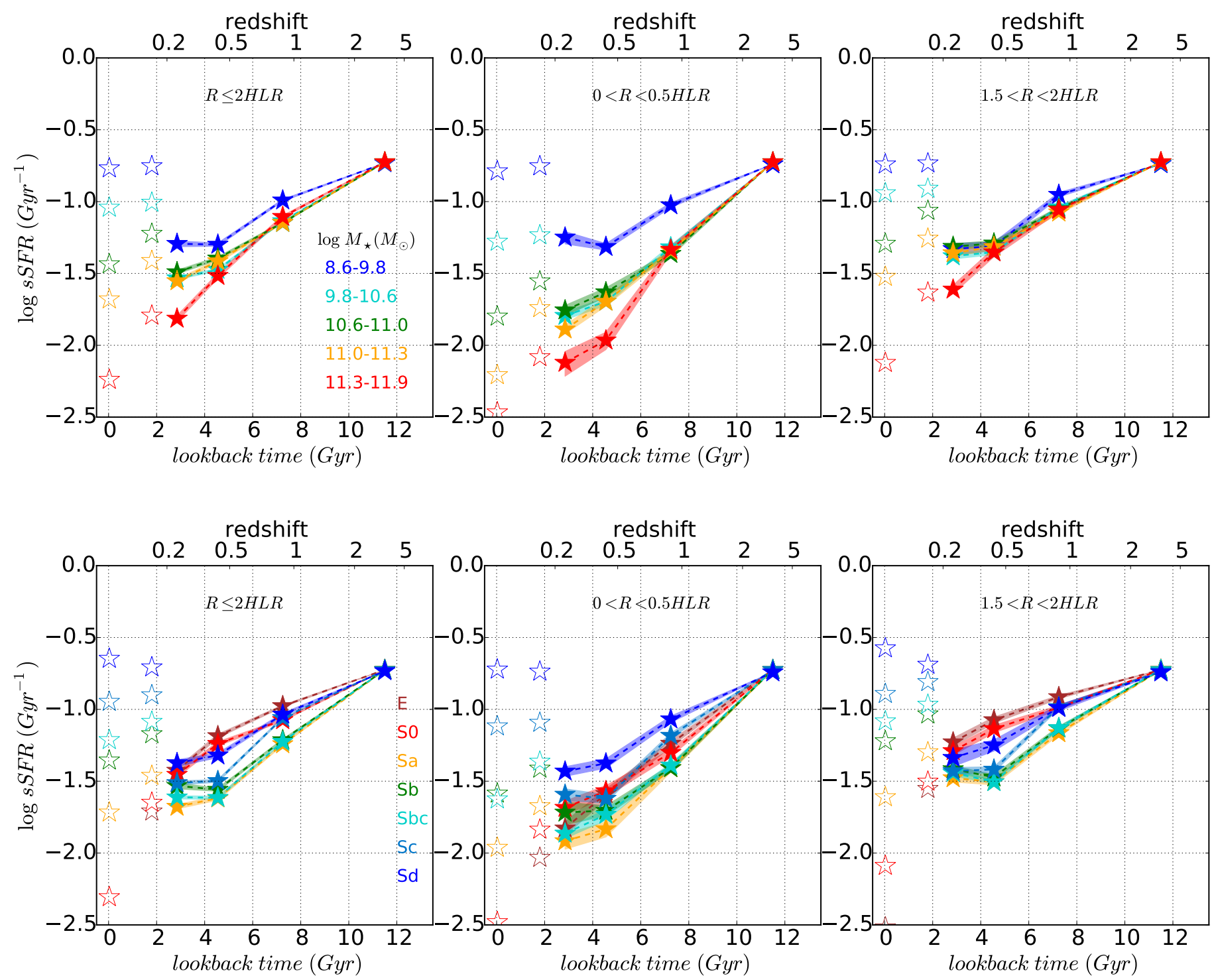

Fig. 7. Same as Fig. 6, but for the specific SFR, defined as the SFR in a time-bin divided by the stellar mass formed up to that time.

galaxies were on the MSSF in those epochs. This similarity is even more evident for regions that are located at distances similar to the region of the solar neighborhood $(1.5<R<2 \mathrm{HLR}$, top right panel), where all the regions follow the same $\operatorname{sSFR}(t)$, independently of $M_{\star}$. By analogy with the result for entire galaxies, we interpret this as evidence that these outer regions were in the past in the local main-sequence relation between $\Sigma_{\mathrm{SFR}}$ and $\mu_{\star}$ (González Delgado et al. 2016; Cano-Díaz et al. 2016). However, conditions are very different in the central regions of galaxies. The top middle panel in Fig. 7 shows that $\operatorname{sSFR}(t)$ declines more rapidly among the most massive galaxies. The inner $R<0.5$ HLR of massive galaxies has been below the local MSSF since $z \sim 0.7$. This may be interpreted as a consequence of an efficient mass-dependent feedback mechanism that quenches star formation more rapidly in galaxies with a larger potential well.

We now turn to the behavior of $\operatorname{sSFR}(t)$ in terms of morphology, as shown in the bottom panels of Fig. 7. At the current epoch, E, S0, and Sa galaxies (all of which are off the MSSF) have $s S F R<0.1 \mathrm{Gyr}^{-1}$. In the inner regions, only late spirals have current sSFRs above $0.1 \mathrm{Gyr}^{-1}$ (although most regions in the disks of spirals have an sSFR above this value). At early epochs, the sSFR in these inner regions shows a similar declining dependence with cosmic time as that seen in the upper panels. We note, however, the remarkable behavior of the outer regions of E and S0 galaxies (bottom right panel): over the $0.4<z<1$ period, the $\operatorname{sSFR}(t)$ curves of these (current epoch) early-type systems run above those of spirals. We hypothesize that this may reflect the growth of the envelope of E and S0 galaxies through mergers.

\subsection{SFHs: Star formation rate intensities}

As a final way of expressing our 2D SFHs, we show results in terms of the surface density of SFR, $\Sigma_{\mathrm{SFR}}$, also referred to as the star formation intensity (SFI). To investigate the time evolution of the SFI, we divide $\mu_{\star}(t)$ (the stellar mass per unit area formed in each epoch, shown in the bottom panels of Fig. 3) by the age span of the corresponding CSP component in the base, obtaining $\Sigma_{\mathrm{SFR}}(t)$. As in the previous section, the information for the last $<2.2 \mathrm{Gyr}$ is lumped into a single bin. We also show the SFI obtained for the last 32 Myr to facilitate comparison with González Delgado et al. (2016), where this shorter timescale was used as a measure of the current $\Sigma_{\mathrm{SFR}}$. Obviously, our data give us no clue on how galaxy sizes and shapes change 

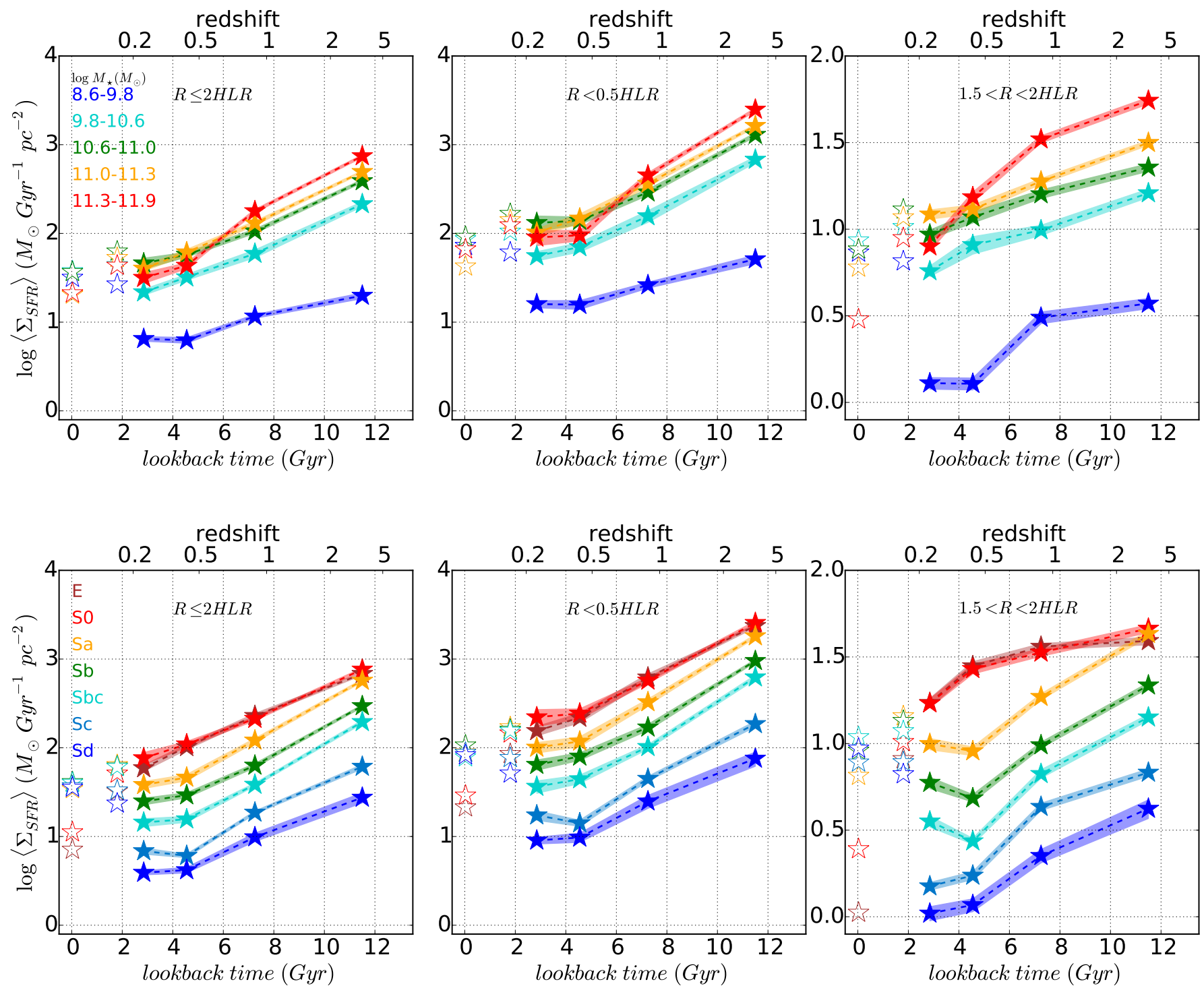

Fig. 8. Same as Fig. 6, but for the star formation intensity, $\Sigma_{\mathrm{SFR}}$.

over time, just as they give no information about where stars that are currently at some location within the galaxy were originally born, both of which affect the actual cosmic evolution of $\Sigma_{\mathrm{SFR}}$. Despite these limitations, SFIs bring valuable insight to this study.

Figure 8 shows the results in the same format as previous figures, with $M_{\star}$ and Hubble-type averages shown in the top and bottom panels, respectively, and right, middle, and left panels showing results for different spatial regions. In general terms, the figure shows that the SFI increases with redshift. This is in line with simple expectations. Gas fractions and densities are both expected and observed to be higher at high $z$ (Tacconi et al. 2013), which, extrapolating from the Schmidt-Kennincutt relation, naturally leads to higher $\Sigma_{\text {SFR }}$ (Barden et al. 2005; Yuma et al. 2011; Förster Schreiber et al. 2011; Mosleh et al. 2012; Carollo et al. 2013). In addition, the smaller galaxy sizes in the past (e.g., van Dokkum et al. 2013) also lead to higher $\Sigma_{\mathrm{SFR}}$, but, as explained above, this effect is not included in our analysis. Higher SFIs are indeed observed in galaxies at high $z$. At $z \sim 2-3, \Sigma_{\mathrm{SFR}} \sim 1000 M_{\odot} \mathrm{Gyr}^{-1} \mathrm{pc}^{-2}$ has been reported by Lehnert et al. (2013), and MW progenitor galaxies are proposed to have $\Sigma_{\mathrm{SFR}} \sim 600 M_{\odot} \mathrm{Gyr}^{-1} \mathrm{pc}^{-2}$ around $z \sim 2$ to explain the formation of a thick disk like the one in the Galaxy (Bovy et al. 2012; Lehnert et al. 2014).

Except for low-mass galaxies, our $\Sigma_{\mathrm{SFR}}$ values increase with redshift by up to $\sim 1.5$ orders of magnitude with respect to values at $z=0$. Our estimates of $\Sigma_{\mathrm{SFR}}$ at $z \geq 2$ averaged over the whole galaxy (left panels) range from $\sim 4000$ to $100 M_{\odot} \mathrm{Gyr}^{-1} \mathrm{pc}^{-2}$ from the highest to the smallest $M_{\star}$ bin. The distribution of $\Sigma_{\mathrm{SFR}}$ with Hubble types ranges from $\sim 4000 M_{\odot} \mathrm{Gyr}^{-1} \mathrm{pc}^{-2}$ in ETGs to $\sim 1000 M_{\odot} \mathrm{Gyr}^{-1} \mathrm{pc}^{-2}$ in Sbc galaxies, reaching a minimum of $150 M_{\odot} \mathrm{Gyr}^{-1} \mathrm{pc}^{-2}$ in Sd galaxies. With the exception of the less massive galaxies $\left(M_{\star}<10^{10}\right)$ and late-type spirals, these values are similar to the $\Sigma_{\mathrm{SFR}}$ observed in high- $z$ galaxies.

The top panels in Fig. 8 show that while at $z=0$ the SFI is nearly independent of $M_{\star}$, in the past, $\Sigma_{\mathrm{SFR}}$ was higher for galaxies that are more massive today. Disregarding the lowest massbin and focusing on our oldest ages, $\Sigma_{\mathrm{SFR}}$ grows by $\sim 0.7 \mathrm{dex}$ for a $\sim 1.5$ dex increase in $M_{\star}$, an approximately $\Sigma_{\text {SFR }} \propto M_{\star}^{0.4-0.5}$ scaling relation. This may be understood in terms of the dependence of galaxy size on mass. Recently, van der Wel et al. (2014) have found that star-forming galaxies with $M_{\star}>3 \times 10^{9}$ have effective radii that for a given redshift grow as $R_{e} \propto M_{\star}^{0.22}$. Similarly, van Dokkum et al. (2013) obtained a $R_{e} \propto M_{\star}^{\star}$ 
size-mass relation (see also Newman et al. 2012; Mosleh et al 2012; Buitrago et al. 2013). Combining this empirical scaling with an $S F R \propto M_{\star}^{0.7-0.9}$ MSSF, and assuming its slope does not vary much at high $z$ (Whitaker et al. 2014), we find $\Sigma_{\mathrm{SFR}} \propto$ $S F R / R_{e}^{2} \propto M^{0.2-0.5}$, which is close to the relation obtained from Fig. 8.

A clear relation between the evolution of $\Sigma_{\mathrm{SFR}}$ and galaxy morphology is revealed in the bottom panels of Fig. 8. At present, all spirals have similar $\Sigma_{\mathrm{SFR}}$ values, well above those of $\mathrm{E}$ and S0 galaxies. This is particularly evident for the estimates over the last $32 \mathrm{Myr}$, but it remains true considering the last Gyr (We note that this difference in current SFI is not as strong in the top panels because of the mixture of morphological types when binning galaxies by $M_{\star}$.) In the past, however, $\Sigma_{\mathrm{SFR}}(t)$ scales with Hubble type, increasing systematically from late to early types. A possible interpretation of this result is that it reflects a sequence in angular momentum, decreasing from early to late types. At high $z$, the combination of low angular momentum and abundant supply of gas favors high gas concentrations and thus, from the Schmidt-Kennicutt law, high SFI. These higher $\Sigma_{\text {SFR }}$ in the past would also imply higher $\mu_{\star}$ if a local MSSF analogous to the one seen today (Wuyts et al. 2013; González Delgado et al. 2016; Cano-Díaz et al. 2016; Maragkoudakis et al. 2017) also existed in the past, so that galaxies that are denser today were also denser in the past.

It is interesting to note that the shape of the $\Sigma_{\mathrm{SFR}}(t)$ curves in the inner regions is similar to the shape of the curves obtained for the whole galaxy, both when binning by $M_{\star}$ (top panels) and by Hubble type (bottom). The same can be said about the outer regions of spirals. E and S0 galaxies, however, behave differently, showing a slower decay in their outer SFI than elsewhere in the galaxy, as can be seen by comparing their $\Sigma_{\mathrm{SFR}}(t)$ curves (in brown and red) in the bottom right panels to the corresponding curves in the left and central panels. We again speculate that this may be signaling the accretion of stars in the envelopes of these galaxies at $z=1-0.5$.

\section{Discussion: SFH constraints on galaxy formation scenarios}

The discovery of high-redshift disk-like galaxies characterized by high-density clumps of star formation (Elmegreen \& Elmegreen 2006; Wuyts et al. 2013; Genzel et al. 2014) have led to the suggestion that mergers at $z>2$ may not be the main mechanism for the formation of progenitors of the current massive galaxies. A possible scenario is that these progenitors had a very rich gaseous disk-like component where high rates of star formation were possible by continuous fueling of cold and filamentary streams of gas from the cosmic web (Kereš et al. 2005; Ocvirk et al. 2008; Dekel \& Burkert 2014). These clumps can arise from gravitational instabilities driven by the continuous replenishment of the disk with high-density gas. If the clumps survive for a long enough time and are not destroyed by internal feedback, dynamical friction could lead them to migrate inward, where they can coalesce to form a compact central component (a "bulge"; Elmegreen et al. 2008).

In galaxies like the MW, where there is no classical bulge (Di Matteo et al. 2014), this early epoch of clump growth can lead to the formation of a turbulent thick disk. The accretion rate would then slow down, the star formation activity decrease, the angular momentum increase, and a thin disk would form.

In this section we try to shed light on some aspects of these scenarios from the perspective of our CALIFA-based 2D
SFH analysis. Since the ideas discussed above pertain to relatively massive galaxies, and also because of the incompleteness of our sample at low masses, we focus the discussion on galaxies with $M_{\star}>10^{10}$

\subsection{Formation of disks in spirals: Comparison of CALIFA SFHs with models for the Milky Way}

Recent modeling of the chemical abundances in the solar vicinity (Haywood et al. 2013, 2015; Snaith et al. 2014, 2015) has shown that the inner disk $(R<10 \mathrm{kpc})$ of the Galaxy has gone through two phases of star formation: (i) the formation of a thick disk, from $t=13$ to 9 Gyr ago; and (ii) the formation of a thin disk, in the last 7 Gyr. Lehnert et al. $(2014,2015)$ and Haywood et al. (2016) extended this analysis, showing that this scenario is useful to explain the formation of MW-like galaxies at high $z$. They proposed that there is a drop in SFI in the thick disk phase to a more quiescent phase at $z<1$, which drop is more significant than the decrease expected from the exhaustion of gas given by a Schmidt-Kennicutt relation. They argued that this cessation of the star formation marks the end of the growth of the thick disk.

Our sample contains several galaxies that are similar to the MW both in terms of mass $\left(M_{\star}=8 \times 10^{10}\right.$, Licquia \& Newman 2015, after converting into the IMF used in our analysis), and morphology ( $\mathrm{Sbc}$ to $\mathrm{Sc}$ ). The spatially and temporally resolved SFRs and SFIs obtained with our analysis thus offer a powerful and independent way of testing the evolutionary scenarios for MW-like galaxies outlined above.

Figure 9 shows the results of this comparison. The left panel compares the evolution of the SFR of the inner disk of the MW as predicted by Haywood et al. (2016), drawn as black solid lines, with the SFR $(t)$ obtained with our analysis for Sbc (circles) and Sc (stars) galaxies in three $M_{\star}$ intervals covering stellar masses similar to that of the Galaxy. Our SFRs are computed for regions within $R<2$ HLR, corresponding to $\sim 10 \mathrm{kpc}$ for Sbc galaxies, and thus matching the size of the MW inner disk (Haywood et al. 2016). In the right panel of Fig. 9 we compare the SFI derived by Lehnert et al. (2015) with our estimates for the same Sbc and Sc galaxies averaging $\Sigma_{\text {SFR }}$ over the central 0.5 HLR, which for these spirals corresponds to the size of the thick disk of the MW (Bland-Hawthorn \& Gerhard 2016).

The similarity of our results for the SFH of Sbc galaxies with $\log M_{\star}=10.7-10.9$ to the $\operatorname{SFR}(t)$ and $\Sigma_{\mathrm{SFR}}(t)$ proposed for the MW is remarkable. Models and data in Fig. 9 both show a significant drop in SFR and its intensity from $z>2$ to $z<1$, with a slight rejuvenation in recent epochs that may be associated with star formation activity in the thin disk. This comparison suggests that the formation of a thick disk can be a common phase early in the life of MW-like galaxies. The similar shapes of the SFHs in the central regions of the late-type spirals in CALIFA (as seen in Figs. 5-8) further suggest that the formation of thick disks 10 Gyr ago is a generic feature in the build-up of these systems.

\subsection{Growth of early-type galaxies}

ATLAS3D has revealed a close link between ETG and spirals by showing that there is a critical dynamical mass of $\sim 2 \times 10^{11}$ below which fast-rotating ETGs form a parallel sequence to spirals in galaxy properties, while slow rotators dominate above this critical mass. Slow rotators have assembled near the center of massive dark matter halos through intense star formation at high redshift, and they remain slow rotators for the rest of their 

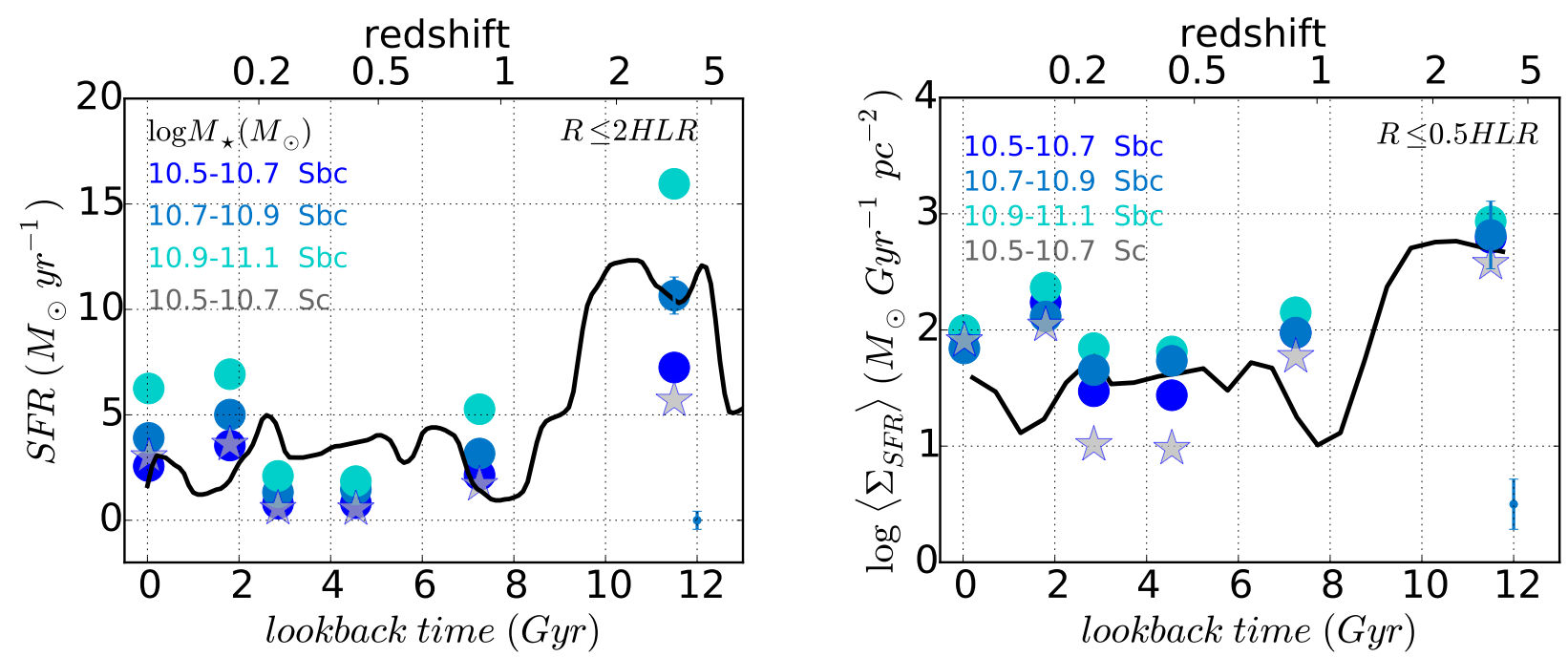

Fig. 9. Evolution of the SFR and SFI for Sbc galaxies (points) in three stellar mass bins (color-coded as indicated in the label), and Sc galaxies (stars) in our lowest $M_{\star}$ bin. The SFR is calculated by adding the spaxels in the inner $R<2$ HLR, while the $\Sigma_{\mathrm{SFR}}$-values are averages over the central 0.5 HLR. The black line shows the model proposed by Haywood et al. (2015) and Lehnert et al. (2014) for high-redshift disk galaxies similar to the MW $\left(\log M_{\star} \sim 10.9\right)$. Bars located at the right side of the plots represent the standard deviations for Sbc galaxies with $10.7 \leq \log M_{\star} \leq 10.9$ at $\sim 11.5 \mathrm{Gyr}$ (upper bar) and the standard deviations for these galaxies averaged for all the epochs (at the right lower corner).

evolution through a channel that is dominated by gas-poor mergers. Fast rotators, on the other hand, start as star-forming disks and evolve through a channel that is dominated by gas accretion, bulge growth, and quenching (Cappellari et al. 2013; Cappellari 2013, 2016).

Using a non-parametric SFH analysis of integrated spectra (up to $R=0.5-1$ HLR for their data) of ATLAS3D galaxies, McDermid et al. (2015) found that ETGs more massive than $M_{\star}=10^{10.5}$ form $90 \%$ of their mass by $z \geq 2$. In contrast, lower mass ETGs have more extended SFHs, forming barely half of their mass before $z=2$.

These results are similar to those obtained here. Figure 8, for instance, indicates that (i) E and S0 in our sample have on average equal SFHs at least during the first 10 Gyr. (ii) In the inner regions, the SFH of E and SO are very similar in shape to the SFH of early-type spirals, except in the last 3-4 Gyr. For instance, the mass fractions at $z>2$ in the inner regions of E and S0 are in the $70-83 \%$ range (depending on $M_{\star}$ ), indistinguishable from the $80 \%$ found for $\mathrm{Sa}$. (iii) In the outer regions, which are not sampled by the ATLAS3D data, the SFH of E and S0 galaxies is quite different from that of $\mathrm{Sa}$ and later types. Between $z=2$ and 0.4 , both $\Sigma_{\mathrm{SFR}}$ and sSFR are significantly higher in ETG than in spirals.

These results indicate that ETG have assembled their inner regions in a similar way to Sa galaxies, probably through gas accretion or mergers and bulge growth. However, their outer regions grow significantly slower than the inner regions, building $40-60 \%$ of their stellar mass over the first few Gyr, compared to $\sim 80 \%$ at $R<0.5 \mathrm{HLR}$. Thus, although the centers of ETG formed very fast and early on, their envelopes were assembled during a more extended period. This epoch of active growth is roughly centered around $z$ of 1 , but it extends from $z \sim 2$ to 0.4 .

It is now well established that massive galaxies $\left(M_{\star}>\right.$ $\left.10^{11}\right)$ at $z \sim 2$ have significantly smaller sizes than their local E and S0 counterparts, and that they have grown significantly since then (Trujillo et al. 2006, 2007; Buitrago et al. 2008; van Dokkum et al. 2010). It has been suggested that dry mergers are the main driver for this late size evolution, expanding their envelope by means of small satellite accretion (Naab et al. 2009; Bell et al. 2004). $z \sim 1$ has been identified as an epoch of galaxy merging (Hammer et al. 2005; Kaviraj et al. 2015), in which the progenitors of ETG increase in size and mass in proportion to one another, approximately following $\Delta \log R_{e} \sim 2 \times \Delta \log M_{\star}$ (van Dokkum et al. 2010, 2015; Huertas-Company et al. 2015). This relation predicts that from $z=2$ to 0.4 , ETGs with a present-day mass of $3 \times 10^{11}$ increase their effective radii from $R_{e} \sim 2.5$ to $6 \mathrm{kpc}$ and their stellar mass by a factor of $\sim 1.5$. Although we cannot test the size evolution with our data, we find that from $z=2$ to 0.4 , our E and S0 galaxies have grown their mass by a factor of 1.5 on average ${ }^{3}$, in excellent agreement with this estimate.

It thus seems that our results for the SFHs of ETGs are in agreement with the two-phase formation scenario for ETGs, where the central part builds most of its mass at high $z$, probably through highly dissipative processes involving gas accretion, while the outer envelope grows over a more extended period (down to $z \sim 0.4$ ), possibly through dry mergers.

\subsection{Cessation of star formation}

High-redshift studies have shown that massive galaxies $\left(M_{\star}>\right.$ $10^{11}$ ) form and quench fast (Cimatti et al. 2004; McCarthy et al. 2004; Whitaker et al. 2013), a scenario that is also supported by studies of local ETGs (McDermid et al. 2015; Citro et al. 2016; Pacifici et al. 2016). We also obtain that most galaxies formed most of their mass early on, but in contrast with these other studies, we find that it took them a long time to complete the cessation of star formation. In other words, we seem to obtain a slower quenching than other studies. There is, however, one case for which we do find evidence for a fast quenching: the most massive ellipticals.

This result appears in several of the previous figures, perhaps most clearly so in Fig. 5, where the steepest SFHs around $z \sim 1$

\footnotetext{
3 This is a global estimate, but our mass growth factors vary with the radial location. Typically, inner regions grow by $20 \%$, while outer regions grow by $60 \%$ in mass over this same period.
} 
R. M. González Delgado et al.: 2D star formation history of CALIFA galaxies
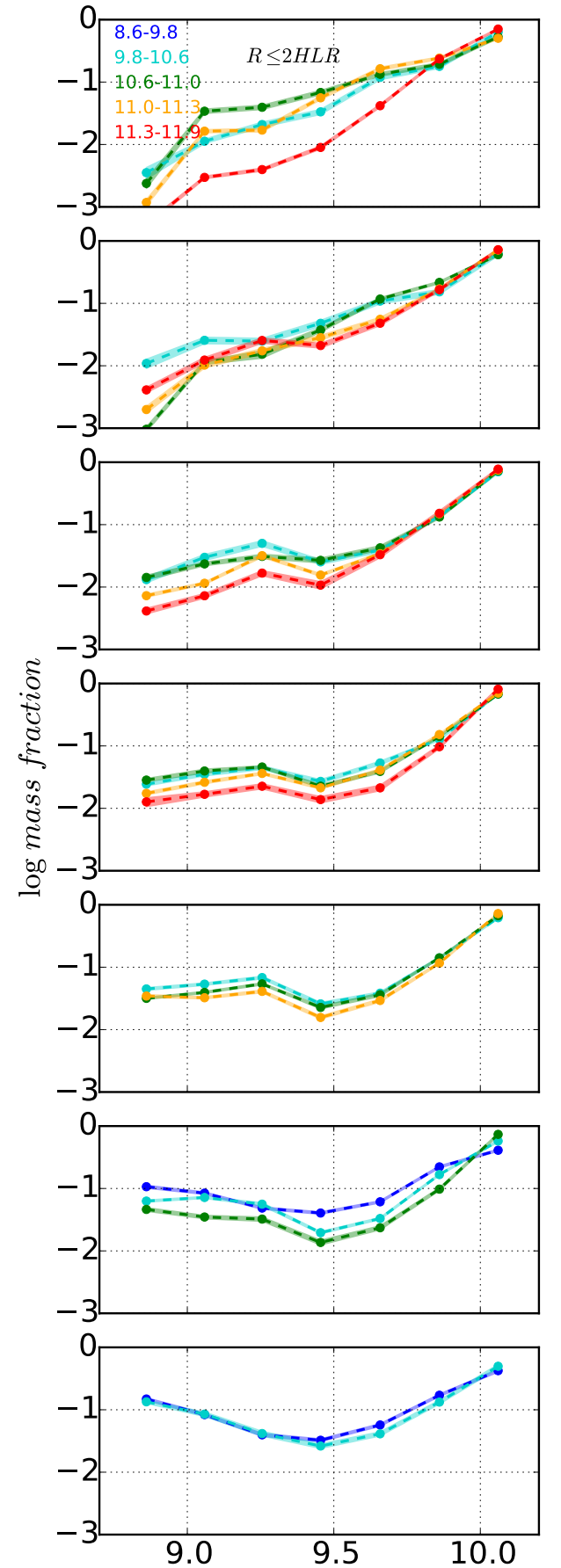
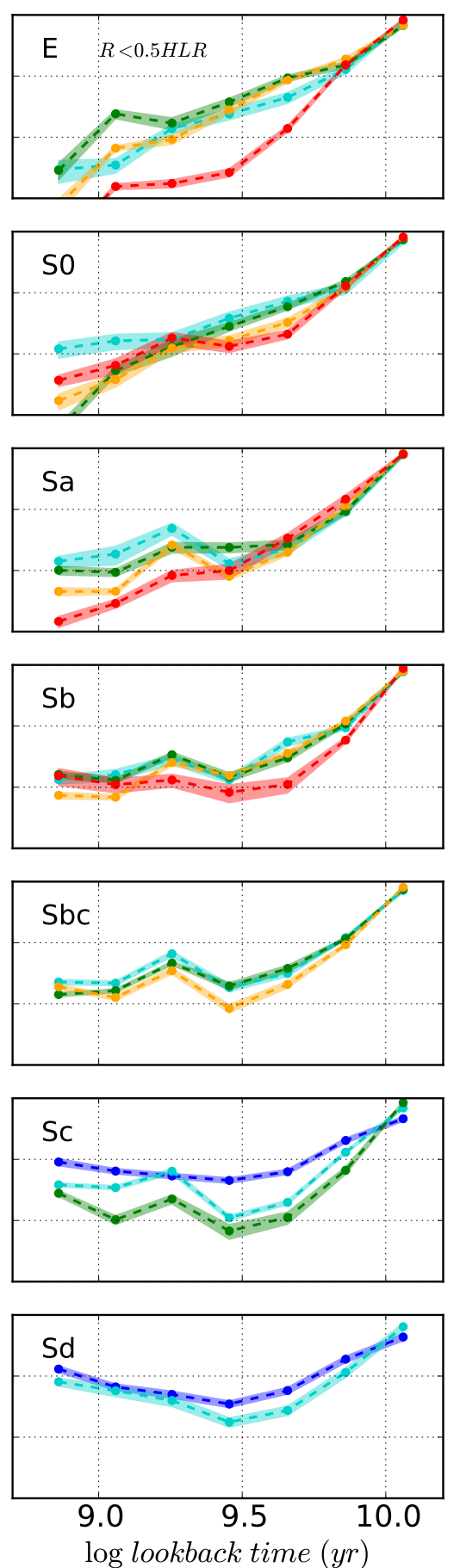
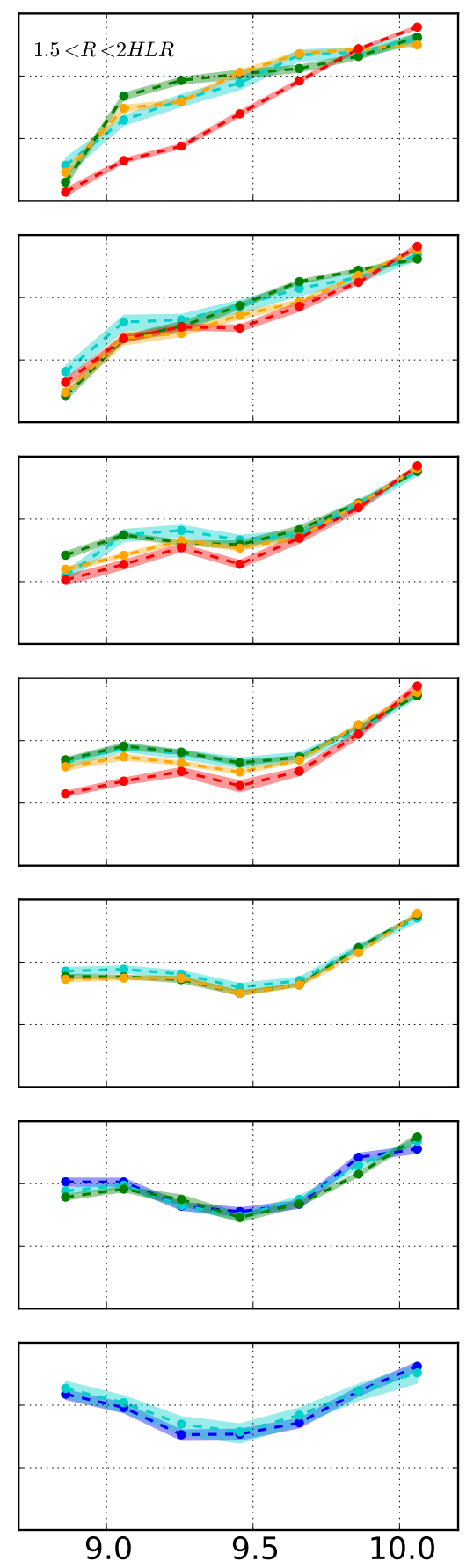

Fig. 10. Same as Fig. 5, but breaking the mass fraction SFHs into bins of Hubble type (E galaxies at the top and Sd at the bottom) and stellar mass (coded by colors, with $M_{\star}$ decreasing from red to blue).

occur for our highest- $M_{\star}$ bin (top panels), or ellipticals (bottom). A close inspection of Fig. 3 shows that this fast quenching occurs not for massive or elliptical galaxies in general, but for galaxies that are both very massive and elliptical. This cannot be fully appreciated in Fig. 5 because its panels collapse over either the $M_{\star}$ or the morphology dimension. To highlight this point and also to separate the effects of $M_{\star}$ and morphology upon SFHs in a visually simpler way, Fig. 10 shows the average mass fraction curves, $m(t)$, for galaxies along the Hubble sequence (E to Sd from top to bottom) for the same $M_{\star}$ bins used throughout the paper (coded by the colors of the curves). The left, middle and right panels separate radial regions in the same order as in Figs. 5-8.

The red curves in the top row of Fig. 10 stand out from all others in being the fastest-declining SFHs in our sample. As pointed out above, this fast quenching is only seen among our most massive ellipticals $\left(\log M_{\star}=11.3-11.9\right)$. S0 and Sa galaxies of the same mass do not have similarly steep SFHs in their first few Gyr of evolution, neither do lower mass ellipticals. This is especially clear in the inner regions (central panels), but it also holds for the galaxy as a whole (left). In all other cases, the decline in star formation activity is slower. 
Assuming that $M_{\star}$ is a good tracer of the halo mass (Behroozi et al. 2013), this result suggests that the halo mass is not the main mechanism in quenching these massive ellipticals. Other studies have suggested that the bias of the dark matter halos depends on something other than their mass, where the initial conditions (e.g., voids, filaments, and geometry of the environment) and halo-assembly history (e.g., halo formation time) are relevant to set the differences in the SFH of galaxies (Dressler et al. 2016) and their galaxy properties (Tojeiro et al. 2017).

\subsection{Red nuggets as galaxy nuclei}

The SFH of many of the nuclei of the galaxies in our sample (central $<0.2 \mathrm{HLR}$, equivalent to $\sim 1 \mathrm{kpc}$ ) show that these central cores formed fast and quenched rapidly. This occurs in ETG and early-type spirals, which in our sample coincide with galaxies more massive than a few $\times 10^{10} M_{\odot}$. Thus, the ETG and Sa-Sb in our sample have formed $>80 \%$ of their central core mass earlier than $z=2$, and with $\Sigma_{\mathrm{SFR}}$ significantly above $10^{3} M_{\odot} \mathrm{pc}^{-2} \mathrm{Gyr}^{-1}$ (up to $5 \times 10^{3} M_{\odot} \mathrm{pc}^{-2} \mathrm{Gyr}^{-1}$ in present-day massive ellipticals). These cores can be a relic of the red nuggets (defined as very compact massive red galaxies that are about five times smaller than equal-mass analogs) detected at high and low redshift (Barro et al. 2013; Ferré-Mateu et al. 2017).

\subsection{Rejuvenation in spirals}

We return to Fig. 10, but now we focus on the more recent past and examine the SFHs for all morphologies. We see that all $\mathrm{E}$ and S0 in our sample experience a further decrease of the star formation in the last $\sim 4 \mathrm{Gyr}$, while in spirals there is a new activation of the star formation over this same period (also seen as the increase in $\Sigma_{\mathrm{SFR}}$ and sSFR in Figs. 7 and 8). This kind of rejuvenation has been also observed in the mass cumulative curve of low-mass galaxies of the extended CALIFA sample (García-Benito et al. 2017), where the mass grows by more than $20 \%$ in the last 2 Gyr. This is also observed by Leitner (2012) in low-mass star-forming galaxies.

This rejuvenation in spirals can be produced by infall of new gas or by the consumption of the residual gas that is already in the galaxy. This phase is clearly less intense in earlythan in late-type spirals, and it is very significant in the outer regions of Sbc-Sc galaxies. It explains why Sbc-Sc-Sd galaxies, and in particular the disk regions, are the main contributor to the present-day star formation rate density of the Universe (González Delgado et al. 2016).

It is well known that the fraction of the mass in $\mathrm{HI}$ increases with both decreasing mass and later type (van Driel et al. 2016), so that such galaxies have an abundance of fuel for forming stars in their disks. The rejuvenation will be stronger in low-mass galaxies $\left(\log M_{\star}<10\right.$ ), where $M_{\mathrm{HI}} / M_{\star} \sim 1$. Thus, they have enough gas if all is used to fuel the galaxy for a mass-doubling time, which is about a Hubble time (see Fig. 7).

However, it is difficult to determine how this can occur. Gravitational torques are probably needed, so it could be that this is related to minor mergers. This is in fact the case in M31, where a small interaction with M32 and M33 or minor mergers are the cause of the global enhancement of star formation in the disk during the last $2-4 \mathrm{Gyr}$, producing $\sim 60 \%$ of the total mass formed in the disk during the last 5 Gyr (Williams et al. 2015). Bars may also act to bring the HI gas from the outer disk and trigger the star formation. This is a plausible mechanism because bars are very frequent in late-type galaxies (Moles et al. 1995; Buta 2013; Buta et al. 2015).

\subsection{Modes of galaxy growth}

Figure 10 also suggests that galaxies can grow in two different modes. For the early evolution of Sa-Sbc spirals and the entire evolution of $\mathrm{E}$ and S0, the logarithm of their mass fraction $(\log m)$ declines with $\log t$. For the later type (Sc-Sd) spirals and low-mass galaxies, $\log m$ is almost constant and independent of time. Thus, one mode is exponential and the other is scale free. This is a feature across all mass and morphological classes, although the balance between the two modes is different.

Perhaps the exponential mode represents the transition between the formation of a thick and a thin disk. The thick disk is a self-regulated mode, where strong outflows and turbulence drive the high intensity of the star formation rate that occurs very early on in the evolution (Lehnert et al. 2015). The thin disk is a scalefree mode regulated by the secular processes; a phase driven by self-gravity, and the energy injection from the stellar population is not relevant for global regulation (Lehnert et al. 2015).

\section{Summary and conclusions}

We have applied the fossil record method of the stellar populations to a sample of 436 galaxies observed by CALIFA at the $3.5 \mathrm{~m}$ telescope in Calar Alto, to investigate their SFH in seven morphology bins (E, S0, Sa, Sb, Sbc, Sc, and Sd) and several stellar masses in the range $\sim 10^{9}$ to $7 \times 10^{11} M_{\odot}$ (for a Salpeter IMF). A full spectral fitting analysis was performed using the STARLIGHT code and a combination of composite stellar populations (CSP) spectra derived with the models of González Delgado et al. (2005) and Vazdekis et al. (2015). This base comprises 18 logarithmically spaced age bins centered at ages from 0.00245 to $11.50 \mathrm{Gyr}$, and eight metallicities from $\log Z / Z_{\odot}=-2.28$ to +0.40 . The spectral fitting results were processed with our PyCASSO pipeline to derive the $2 \mathrm{D}(R \times t)$ map of the SFH for each galaxy, from which we obtained the spatial and temporal evolution information of the SFR, sSFR, and the $\Sigma_{\mathrm{SFR}}$.

Our main results are listed below.

1. These nearby galaxies formed very fast. All of them have their peak star formation at the earliest time $(z>2)$, independently of their stellar mass. However, the subsequent SFH varies with $M_{\star}$, with less massive galaxies showing a longer period of star formation. This is a manifestation of the "downsizing" effect observed in other surveys.

2. SFRs decline rapidly as the Universe evolves; at any epoch, the SFR is proportional to $M_{\star}$, and the most massive galaxies had the highest absolute SFR. The sSFR also decreases with time. The $\operatorname{sSFR}(t)$ curves vary systematically with $M_{\star}$ in the central regions, but not in the outer disk of spirals, nor in the envelopes of ellipticals. At the current epoch, sSFR $\geq$ $0.1 \mathrm{Gyr}^{-1}$ for galaxies that are in the MSSF and in regions located in the disk of spirals of Hubble-type later than Sa.

3. The $\Sigma_{\mathrm{SFR}}$ also declines rapidly as the Universe evolves. At the current epoch, the spatially averaged $\Sigma_{\mathrm{SFR}}$ is similar in all spirals $\left(\sim 37 M_{\odot} \mathrm{Gyr}^{-1} \mathrm{pc}^{-2}\right)$, significantly higher than in ETGs. In the past, however, $\Sigma_{\text {SFR }}$ increases systematically from late to early Hubble types. The highest values are found among the progenitors of present-day E and S0 galaxies, with $\Sigma_{\mathrm{SFR}} \sim 10^{3} M_{\odot} \mathrm{Gyr}^{-1} \mathrm{pc}^{-2}$ at $z>2$. These values are similar to those reported for high-redshift star-forming galaxies. 
R. M. González Delgado et al.: 2D star formation history of CALIFA galaxies

4. There is a remarkable similarity between the SFH of Sbc galaxies of $M_{\star} \sim 7 \times 10^{10} M_{\odot}$ in our sample and the predictions for MW-like galaxies proposed by Haywood et al. (2015) and Lehnert et al. (2015) for the formation of the thick disk. In agreement with these models, we obtain that in the central $\left(R<0.5\right.$ HLR) $\Sigma_{\text {SFR }}$ shows a significant drop from $600 M_{\odot} \mathrm{Gyr}^{-1} \mathrm{pc}^{-2}$ at $z>2$ to $100 M_{\odot} \mathrm{Gyr}^{-1} \mathrm{pc}^{-2}$ at $z<1$, while the global SFR decreases from $\sim 10$ to $2 M_{\odot} \mathrm{yr}^{-1}$ over the same period. These comparisons suggest that the formation of a thick disk may be a common phase early on in the life of late-type spirals.

5. In regions located in the envelope of $\mathrm{E}$ and $\mathrm{S} 0$ (akin to $1.5<R<2$ HLR in Figs. 5-8), the mass fraction, SFR, sSFR, and $\Sigma_{\text {SFR }}$ decline with redshift $(2>z>0.4)$ more slowly than regions in the disk of early spirals. The central cores $(R<0.5 \mathrm{HLR})$ have grown their mass very early $(70 \%$ and $83 \%$ at $z>2$ in E and S0 galaxies, respectively), and only increase their mass by a factor 1.2 between $0.4<z<2$, while the outer regions have increased their mass by a factor $\sim 1$.6. These results suggest that $z$ around 1 is an active epoch of envelope assembly in E and S0, in line with the expectations of the two-phase scenario proposed for the formation of ETG.

6. No fast quenching is observed in the SFH of most of the CALIFA galaxies. One exception occurs in the most massive $\left(M_{\star}>10^{11.3} M_{\odot}\right)$ E galaxies, in which SFR and $\Sigma_{\mathrm{SFR}}$ decline very rapidly; in particular, in the central region where most of the mass formed at $z>2$, and the sSFR drops suddenly after $z=1$. However, this fast quenching does not occur in galaxies of the same stellar mass but later type ( $\mathrm{S} 0$ and $\mathrm{Sa}$ in our sample). These results suggest that halo quenching is not the main driver for the cessation of star formation in these galaxies.

7. The previous item notwithstanding, the SFH of most nuclei $(\sim 1 \mathrm{kpc})$ in these galaxies formed fast and quenched rapidly. They formed more than $80 \%$ of their mass at $z>2$, when $\Sigma_{\mathrm{SFR}}>10^{3} M_{\odot} \mathrm{Gyr}^{-1} \mathrm{pc}^{-2}$. They can be the relic of the "red nuggets" detected in high-redshift galaxies.

In summary, the SFH of nearby spirals as represented by the CALIFA survey are compatible with a scenario of fast formation and a relatively long declining phase for the cessation of star formation, which may be sustained by the slow consumption of residual gas from the initial flow. A reactivation of the star formation occurs in more recent times, the last $4 \mathrm{Gyr}$, with an intensity significantly below the peak values, producing a rejuvenation of the disks that is significantly more relevant in lowmass and late spiral types than in more massive and early-type spirals. In ETGs, the initial phases in the formation are similar to those in massive early-type spirals, but E and S0 also have an active long phase of growth between $0.4<z<2$ that is relevant for the growth of their external envelope.

Acknowledgements. CALIFA is the first legacy survey carried out at Calar Alto. The CALIFA collaboration would like to thank the IAA-CSIC and MPIA-MPG as major partners of the observatory, and CAHA itself, for the unique access to telescope time and support in manpower and infrastructures. We also thank the CAHA staff for the dedication to this project. Support from the Spanish Ministerio de Economía y Competitividad, through projects AYA2016-77846-P, AYA2014-57490-P, AYA2010-15081, and Junta de Andalucía FQ1580, AYA2010-22111-C03-03, AYA2010-10904E, AYA201342227P, RyC-2011-09461, AYA2013-47742-C4-3-P, EU SELGIFS exchange programme FP7-PEOPLE-2013-IRSES-612701, CONACYT-125180, DGAPAIA100815, and PAPIIT-DGAPA-IA101217. We also thank the Viabilidad, Diseño, Acceso y Mejora funding program, ICTS-2009-10, for funding the data acquisition of this project. A.L.d.A., E.A.D.L. and R.C.F. thanks for the hospitality of the IAA and the support of CNPq. R.G.D. acknowledges the support of CNPq
(Brazil) through Programa Ciência sem Fronteiras (401452/2012-3). C.J.W. acknowledges support through the Marie Curie Career Integration Grant 303912. We acknowledge the support of the IAA Computing group, to Dra. Marino, and the referee for useful comments.

\section{References}

Abramson, L. E., Gladders, M. D., Dressler, A., et al. 2016, ApJ, 832, 7 Asari, N. V., Cid Fernandes, R., Stasińska, G., et al. 2007, MNRAS, 381, 263 Baldry, I. K., Glazebrook, K., Brinkmann, J., et al. 2004, ApJ, 600, 681 Barden, M., Rix, H.-W., Somerville, R. S., et al. 2005, ApJ, 635, 959 Barro, G., Faber, S. M., Pérez-González, P. G., et al. 2013, ApJ, 765, 104 Behroozi, P. S., Wechsler, R. H., \& Conroy, C. 2013, ApJ, 770, 57 Bekeraitè, S., Walcher, C. J., Wisotzki, L., et al. 2016, ApJ, 827, L36 Belfiore, F., Maiolino, R., Maraston, C., et al. 2017, MNRAS, 466, 2570 Bell, E. F., McIntosh, D. H., Barden, M., et al. 2004, ApJ, 600, L11 Bell, E. F., Zheng, X. Z., Papovich, C., et al. 2007, ApJ, 663, 834 Bernard, E. J., Ferguson, A. M. N., Richardson, J. C., et al. 2015, MNRAS, 446, 2789

Birnboim, Y., Dekel, A., \& Neistein, E. 2007, MNRAS, 380, 339

Bland-Hawthorn, J., \& Gerhard, O. 2016, ARA\&A, 54, 529

Blanton, M. R., \& Moustakas, J. 2009, ARA\&A, 47, 159

Blanton, M. R., Hogg, D. W., Bahcall, N. A., et al. 2003, ApJ, 592, 819

Bovy, J., Rix, H.-W., \& Hogg, D. W. 2012, ApJ, 751, 131

Brinchmann, J., Charlot, S., White, S. D. M., et al. 2004, MNRAS, 351, 1151

Bruzual A., G., \& Kron, R. G. 1980, ApJ, 241, 25

Bryant, J. J., Owers, M. S., Robotham, A. S. G., et al. 2015, MNRAS, 447, 2857 Buitrago, F., Trujillo, I., Conselice, C. J., et al. 2008, ApJ, 687, L61

Buitrago, F., Trujillo, I., Conselice, C. J., \& Häußler, B. 2013, MNRAS, 428, 1460

Bundy, K., Bershady, M. A., Law, D. R., et al. 2015, ApJ, 798, 7

Buta, R. J. 2013, in Secular Evolution of Galaxies, eds. J. Falcón-Barroso, \& J. H. Knapen, 155

Buta, R. J., Sheth, K., Athanassoula, E., et al. 2015, ApJS, 217, 32

Cano-Díaz, M., Sánchez, S. F., Zibetti, S., et al. 2016, ApJ, 821, L26 Cappellari, M. 2013, ApJ, 778, L2

Cappellari, M. 2016, ARA\&A, 54, 597

Cappellari, M., \& Copin, Y. 2003, MNRAS, 342, 345

Cappellari, M., Emsellem, E., Krajnović, D., et al. 2011, MNRAS, 413, 813

Cappellari, M., McDermid, R. M., Alatalo, K., et al. 2013, MNRAS, 432, 1862

Cardelli, J. A., Clayton, G. C., \& Mathis, J. S. 1989, ApJ, 345, 245

Carollo, C. M., Bschorr, T. J., Renzini, A., et al. 2013, ApJ, 773, 112

Cassarà, L. P., Maccagni, D., Garilli, B., et al. 2016, A\&A, 593, A9

Catalán-Torrecilla, C., Gil de Paz, A., Castillo-Morales, A., et al. 2015, A\&A, 584, A87

Charbonnel, C., Meynet, G., Maeder, A., Schaller, G., \& Schaerer, D. 1993, A\&AS, 101, 415

Cid Fernandes, R., Mateus, A., Sodré, L., Stasińska, G., \& Gomes, J. M. 2005, MNRAS, 358, 363

Cid Fernandes, R., Pérez, E., García Benito, R., et al. 2013, A\&A, 557, A86

Cimatti, A., Daddi, E., Renzini, A., et al. 2004, Nature, 430, 184

Citro, A., Pozzetti, L., Moresco, M., \& Cimatti, A. 2016, A\&A, 592, A19

Cordier, D., Pietrinferni, A., Cassisi, S., \& Salaris, M. 2007, AJ, 133, 468

Croton, D. J., Springel, V., White, S. D. M., et al. 2006, MNRAS, 365, 11

Daddi, E., Dickinson, M., Morrison, G., et al. 2007, ApJ, 670, 156

de Amorim, A. L., García-Benito, R., Cid Fernandes, R., et al. 2017, MNRAS, 471,3727

Dekel, A., \& Birnboim, Y. 2006, MNRAS, 368, 2

Dekel, A., \& Burkert, A. 2014, MNRAS, 438, 1870

Dekel, A., Birnboim, Y., Engel, G., et al. 2009, Nature, 457, 451

Di Matteo, P., Haywood, M., Gómez, A., et al. 2014, A\&A, 567, A122

Di Matteo, T., Springel, V., \& Hernquist, L. 2005, Nature, 433, 604

Dopita, M. A., \& Ryder, S. D. 1994, ApJ, 430, 163

Dressler, A., Kelson, D. D., Abramson, L. E., et al. 2016, ApJ, 833, 251

Dutton, A. A., van den Bosch, F. C., \& Dekel, A. 2010, MNRAS, 405, 1690

Elbaz, D., Daddi, E., Le Borgne, D., et al. 2007, A\&A, 468, 33

Elbaz, D., Dickinson, M., Hwang, H. S., et al. 2011, A\&A, 533, A119

Elmegreen, B. G., \& Elmegreen, D. M. 2006, ApJ, 650, 644

Elmegreen, B. G., Bournaud, F., \& Elmegreen, D. M. 2008, ApJ, 688, 67

Faber, S. M., Willmer, C. N. A., Wolf, C., et al. 2007, ApJ, 665, 265

Fardal, M. A., Katz, N., Weinberg, D. H., \& Davé, R. 2007, MNRAS, 379, 985

Ferré-Mateu, A., Trujillo, I., Martín-Navarro, I., et al. 2017, MNRAS, 467, 1929

Feulner, G., Gabasch, A., Salvato, M., et al. 2005, ApJ, 633, L9

Förster Schreiber, N. M., Shapley, A. E., Erb, D. K., et al. 2011, ApJ, 731, 65

Gallagher, III, J. S., Hunter, D. A., \& Tutukov, A. V. 1984, ApJ, 284, 544 
Gallazzi, A., Bell, E. F., Zibetti, S., Brinchmann, J., \& Kelson, D. D. 2014, ApJ, 788,72

Gallazzi, A., Charlot, S., Brinchmann, J., White, S. D. M., \& Tremonti, C. A. 2005, MNRAS, 362, 41

García-Benito, R., Zibetti, S., Sánchez, S. F., et al. 2015, A\&A, 576, A135

García-Benito, R., González Delgado, R. M., Pérez, E., et al. 2017, A\&A, in press, DOI: 10.1051/0004-6361/201731357

Genzel, R., Förster Schreiber, N. M., Lang, P., et al. 2014, ApJ, 785, 75

Girardi, L., Bressan, A., Bertelli, G., \& Chiosi, C. 2000, A\&AS, 141, 371

Gladders, M. D., Oemler, A., Dressler, A., et al. 2013, ApJ, 770, 64

Goddard, D., Thomas, D., Maraston, C., et al. 2017, MNRAS, 466, 4731

González Delgado, R. M., Cerviño, M., Martins, L. P., Leitherer, C., \& Hauschildt, P. H. 2005, MNRAS, 357, 945

González Delgado, R. M., Cid Fernandes, R., García-Benito, R., et al. 2014a, ApJ, 791, L16

González Delgado, R. M., Pérez, E., Cid Fernandes, R., et al. 2014b, A\&A, 562, A47

González Delgado, R. M., García-Benito, R., Pérez, E., et al. 2015, A\&A, 581, A103

González Delgado, R. M., Cid Fernandes, R., Pérez, E., et al. 2016, A\&A, 590, A44

Hammer, F., Flores, H., Elbaz, D., et al. 2005, A\&A, 430, 115

Haywood, M., Di Matteo, P., Lehnert, M. D., Katz, D., \& Gómez, A. 2013, A\&A 560, A109

Haywood, M., Di Matteo, P., Snaith, O., \& Lehnert, M. D. 2015, A\&A, 579, A5

Haywood, M., Lehnert, M. D., Di Matteo, P., et al. 2016, A\&A, 589, A66

Heavens, A., Panter, B., Jimenez, R., \& Dunlop, J. 2004, Nature, 428, 625

Hopkins, A. M., \& Beacom, J. F. 2006, ApJ, 651, 142

Huang, M.-L., Kauffmann, G., Chen, Y.-M., et al. 2013, MNRAS, 431, 2622

Huertas-Company, M., Pérez-González, P. G., Mei, S., et al. 2015, ApJ, 809, 95

Husemann, B., Jahnke, K., Sánchez, S. F., et al. 2013, A\&A, 549, A87

Ibarra-Medel, H. J., Sánchez, S. F., Avila-Reese, V., et al. 2016, MNRAS, 463, 2799

Karim, A., Schinnerer, E., Martínez-Sansigre, A., et al. 2011, ApJ, 730, 61

Kauffmann, G., Heckman, T. M., White, S. D. M., et al. 2003, MNRAS, 341, 54

Kaviraj, S., Devriendt, J., Dubois, Y., et al. 2015, MNRAS, 452, 2845

Kelz, A., Verheijen, M. A. W., Roth, M. M., et al. 2006, PASP, 118, 129

Kennicutt, Jr., R. C. 1998, ARA\&A, 36, 189

Kereš, D., Katz, N., Weinberg, D. H., \& Davé, R. 2005, MNRAS, 363, 2

Koleva, M., Prugniel, P., Bouchard, A., \& Wu, Y. 2009, A\&A, 501, 1269

Koleva, M., Prugniel, P., de Rijcke, S., \& Zeilinger, W. W. 2011, MNRAS, 417, 1643

Law, D. R., Yan, R., Bershady, M. A., et al. 2015, AJ, 150, 19

Lehnert, M. D., Le Tiran, L., Nesvadba, N. P. H., et al. 2013, A\&A, 555, A72

Lehnert, M. D., Di Matteo, P., Haywood, M., \& Snaith, O. N. 2014, ApJ, 789 L 30

Lehnert, M. D., van Driel, W., Le Tiran, L., Di Matteo, P., \& Haywood, M. 2015, A\&A, 577, A112

Leitner, S. N. 2012, ApJ, 745, 149

Licquia, T. C., \& Newman, J. A. 2015, ApJ, 806, 96

Lilly, S. J., Le Fevre, O., Hammer, F., \& Crampton, D. 1996, ApJ, 460, L1

Lilly, S. J., Carollo, C. M., Pipino, A., Renzini, A., \& Peng, Y. 2013, ApJ, 772, 119

Madau, P., \& Dickinson, M. 2014, ARA\&A, 52, 415

Madau, P., Pozzetti, L., \& Dickinson, M. 1998, ApJ, 498, 106

Magdis, G. E., Elbaz, D., Daddi, E., et al. 2010, ApJ, 714, 1740

Man, A. W. S., Toft, S., Zirm, A. W., Wuyts, S., \& van der Wel, A. 2012, ApJ, 744,85

Maragkoudakis, A., Zezas, A., Ashby, M. L. N., \& Willner, S. P. 2017, MNRAS, 466, 1192

Maraston, C., Pforr, J., Renzini, A., et al. 2010, MNRAS, 407, 830

Maraston, C., Pforr, J., Henriques, B. M., et al. 2013, MNRAS, 435, 276

Martig, M., Bournaud, F., Teyssier, R., \& Dekel, A. 2009, ApJ, 707, 250

Mateus, A., Sodré, L., Cid Fernandes, R., et al. 2006, MNRAS, 370, 721

McCarthy, P. J., Le Borgne, D., Crampton, D., et al. 2004, ApJ, 614, L9

McDermid, R. M., Alatalo, K., Blitz, L., et al. 2015, MNRAS, 448, 3484

Moles, M., Marquez, I., \& Perez, E. 1995, ApJ, 438, 604

Mosleh, M., Williams, R. J., Franx, M., et al. 2012, ApJ, 756, L12

Naab, T., \& Ostriker, J. P. 2016, ARA\&A, 55, 59

Naab, T., Johansson, P. H., \& Ostriker, J. P. 2009, ApJ, 699, L178

Neistein, E., \& Dekel, A. 2008, MNRAS, 388, 1792

Neistein, E., van den Bosch, F. C., \& Dekel, A. 2006, MNRAS, 372, 933

Newman, A. B., Ellis, R. S., Bundy, K., \& Treu, T. 2012, ApJ, 746, 162

Noeske, K. G., Faber, S. M., Weiner, B. J., et al. 2007, ApJ, 660, L47

Ocvirk, P., Pichon, C., Lançon, A., \& Thiébaut, E. 2006, MNRAS, 365, 74

Ocvirk, P., Pichon, C., \& Teyssier, R. 2008, MNRAS, 390, 1326

Oemler, Jr., A., Dressler, A., Gladders, M. G., et al. 2013, ApJ, 770, 63
Oliver, S., Frost, M., Farrah, D., et al. 2010, MNRAS, 405, 2279 Pacifici, C., Kassin, S. A., Weiner, B. J., et al. 2016, ApJ, 832, 79 Panter, B., Heavens, A. F., \& Jimenez, R. 2003, MNRAS, 343, 1145 Panter, B., Jimenez, R., Heavens, A. F., \& Charlot, S. 2008, MNRAS, 391, 1117

Papovich, C., Finkelstein, S. L., Ferguson, H. C., Lotz, J. M., \& Giavalisco, M. 2011, MNRAS, 412, 1123

Pérez, E., Cid Fernandes, R., González Delgado, R. M., et al. 2013, ApJ, 764, L1 Pietrinferni, A., Cassisi, S., Salaris, M., \& Castelli, F. 2004, ApJ, 612, 168 Pietrinferni, A., Cassisi, S., Salaris, M., \& Castelli, F. 2006, ApJ, 642, 797 Pietrinferni, A., Cassisi, S., Salaris, M., Percival, S., \& Ferguson, J. W. 2009, ApJ, 697, 275

Pietrinferni, A., Cassisi, S., Salaris, M., \& Hidalgo, S. 2013, A\&A, 558, A46

Renzini, A., \& Peng, Y.-j. 2015, ApJ, 801, L29

Roberts, M. S., \& Haynes, M. P. 1994, ARA\&A, 32, 115

Rodighiero, G., Vaccari, M., Franceschini, A., et al. 2010, A\&A, 515, A8

Roth, M. M., Kelz, A., Fechner, T., et al. 2005, PASP, 117, 620

Salim, S., Rich, R. M., Charlot, S., et al. 2007, ApJS, 173, 267

Sánchez, S. F., García-Benito, R., Zibetti, S., et al. 2016, A\&A, 594, A36

Sánchez, S. F., Kennicutt, R. C., Gil de Paz, A., et al. 2012, A\&A, 538, A8

Sánchez Almeida, J., Elmegreen, B. G., Muñoz-Tuñón, C., \& Elmegreen, D. M. 2014, A\&A Rev., 22, 71

Sánchez-Blázquez, P., Peletier, R. F., Jiménez-Vicente, J., et al. 2006, MNRAS, 371,703

Sánchez-Blázquez, P., Rosales-Ortega, F. F., Méndez-Abreu, J., et al. 2014, A\&A, 570, A6

Sandage, A. 1986, A\&A, 161, 89

Scalo, J. M. 1986, Fund. Cosmic Phys., 11, 1

Schaerer, D., Charbonnel, C., Meynet, G., Maeder, A., \& Schaller, G. 1993, A\&AS, 102, 339

Schaller, G., Schaerer, D., Meynet, G., \& Maeder, A. 1992, A\&AS, 96, 269

Schiminovich, D., Wyder, T. K., Martin, D. C., et al. 2007, ApJS, 173, 315

Searle, L., Sargent, W. L. W., \& Bagnuolo, W. G. 1973, ApJ, 179, 427

Shi, Y., Helou, G., Yan, L., et al. 2011, ApJ, 733, 87

Snaith, O., Haywood, M., Di Matteo, P., et al. 2015, A\&A, 578, A87

Snaith, O. N., Haywood, M., Di Matteo, P., et al. 2014, ApJ, 781, L31

Speagle, J. S., Steinhardt, C. L., Capak, P. L., \& Silverman, J. D. 2014, ApJS, 214,15

Stark, D. P., Schenker, M. A., Ellis, R., et al. 2013, ApJ, 763, 129

Tacchella, S., Carollo, C. M., Renzini, A., et al. 2015, Science, 348, 314

Tacchella, S., Dekel, A., Carollo, C. M., et al. 2016, MNRAS, 458, 242

Tacconi, L. J., Neri, R., Genzel, R., et al. 2013, ApJ, 768, 74

Tinsley, B. M. 1968, ApJ, 151, 547

Tinsley, B. M. 1972, A\&A, 20, 383

Tojeiro, R., Heavens, A. F., Jimenez, R., \& Panter, B. 2007, MNRAS, 381, 1252

Tojeiro, R., Wilkins, S., Heavens, A. F., Panter, B., \& Jimenez, R. 2009, ApJS, 185,1

Tojeiro, R., Percival, W. J., Heavens, A. F., \& Jimenez, R. 2011, MNRAS, 413, 434

Tojeiro, R., Eardley, E., Peacock, J. A., et al. 2017, MNRAS, 470, 3720 Trujillo, I., Förster Schreiber, N. M., Rudnick, G., et al. 2006, ApJ, 650, 18

Trujillo, I., Conselice, C. J., Bundy, K., et al. 2007, MNRAS, 382, 109 van der Wel, A., Franx, M., van Dokkum, P. G., et al. 2014, ApJ, 788, 28 van Dokkum, P. G., Whitaker, K. E., Brammer, G., et al. 2010, ApJ, 709, 1018 van Dokkum, P. G., Leja, J., Nelson, E. J., et al. 2013, ApJ, 771, L35 van Dokkum, P. G., Nelson, E. J., Franx, M., et al. 2015, ApJ, 813, 23 van Driel, W., Butcher, Z., Schneider, S., et al. 2016, A\&A, 595, A118 Vazdekis, A., Sánchez-Blázquez, P., Falcón-Barroso, J., et al. 2010, MNRAS, 404, 1639

Vazdekis, A., Coelho, P., Cassisi, S., et al. 2015, MNRAS, 449, 1177

Verheijen, M. A. W., Bershady, M. A., Andersen, D. R., et al. 2004, Astron. Nachr., 325, 151

Walcher, C. J., Lamareille, F., Vergani, D., et al. 2008, A\&A, 491, 713

Walcher, C. J., Wisotzki, L., Bekeraité, S., et al. 2014, A\&A, 569, A1

Whitaker, K. E., van Dokkum, P. G., Brammer, G., \& Franx, M. 2012, ApJ, 754, L29

Whitaker, K. E., van Dokkum, P. G., Brammer, G., et al. 2013, ApJ, 770, L39

Whitaker, K. E., Franx, M., Leja, J., et al. 2014, ApJ, 795, 104

Williams, B. F., Dalcanton, J. J., Dolphin, A. E., et al. 2015, ApJ, 806, 48

Williams, R. J., Quadri, R. F., \& Franx, M. 2011, ApJ, 738, L25

Worthey, G. 1994, ApJS, 95, 107

Wuyts, S., Förster Schreiber, N. M., van der Wel, A., et al. 2011, ApJ, 742, 96

Wuyts, S., Förster Schreiber, N. M., Nelson, E. J., et al. 2013, ApJ, 779, 135

Yuma, S., Ohta, K., Yabe, K., Kajisawa, M., \& Ichikawa, T. 2011, ApJ, 736, 92

Zheng, Z., Wang, H., Ge, J., et al. 2017, MNRAS, 465, 4572

Zibetti, S., Gallazzi, A. R., Ascasibar, Y., et al. 2017, MNRAS, 468, 1902 


\section{Appendix A: Dependence of the stellar population properties on CSP models}

To evaluate to which extent our results depend on the choice of the CSPs instead of the SSPs, we compared the properties derived with two sets of bases: a) CSPs, which are used in the main text and are described in Sect. 3; and b) SSPs, built in a similar way as the base GMe (e.g., González Delgado et al. 2014b), but now using Vazdekis et al. (2015) instead of Vazdekis et al. (2010). This new SSP base is a combination of 254 SSPs, with eight metallicities from $\log Z / Z_{\odot}=-2.28$ to +0.40 , the same as our CSP base. The age is sampled by 37 SSPs per metallicity covering from 1 Myr to 14 Gyr. Like our CSPs, these SSPs, with $t>63 \mathrm{Myr}$, are based on models that match the Galactic abundance pattern imprinted in the MILES stars (Sánchez-Blázquez et al. 2006). In both bases, the IMF is that of Salpeter, and the dust effects are modeled as a foreground screen with a Cardelli et al. (1989) reddening law. More details of this base are described in García-Benito et al. (2017).

Using our pipeline PyCASSO, we obtained the radial distribution of the stellar population properties for each galaxy with a spatial sampling of 0.1 HLR. Figure A.1, panels (b) to (l), compares the results obtained with the CSPs ( $y$-axis) and with the SSPs ( $x$-axis) for a total of 8720 points corresponding to a maximum of 20 radial points (from the nucleus to 2 HLR), for each of the 436 galaxies analyzed in this work. Each panel quotes the mean $\bar{\Delta}=\operatorname{property}(\mathrm{CSP})-\operatorname{property}(\mathrm{SPS})$, and the standard deviation $(\sigma)$. The color indicates the density of points in a logarithmic scale. Panel (a) in Fig. A.1 compares $M_{\star}$ with the two bases, with galaxies colored by their Hubble type.

On average, both methods provide similar results. There are no significant differences between the two sets of results for $M_{\star}$ and for $\mu_{\star}$, with $\bar{\Delta} \sim 0$ and $\sigma \sim 0.03$ dex. Other properties, such as $\tau_{V}$, light-weighted age $\langle\log t\rangle_{L}$, mass-weighted age $\langle\log t\rangle_{M}$, and mass-weighted metallicity $\langle\log Z\rangle_{M}$, are also very similar, with $\bar{\Delta}(\sigma)=0.01(0.03),-0.04(0.08),-0.03(0.05)$, and $0.05(0.08)$, respectively. A slight age-metallicity degeneracy is seen here; CSP models tend to obtain slightly younger ages and higher metallicities. Thus, the spectral fits with CSPs and SSPs provide equal average stellar population properties in galaxies.

To determine the differences in the SFH, we plot in Fig. A.1 the light ( $x$, panels in the third column) and mass ( $m$, panels in the fourth column) fractions compressed into three ( $t \leq 1 \mathrm{Gyr}$, $4<t \leq 9 \mathrm{Gyr}$, and $t>9 \mathrm{Gyr}$ ) of the four representative lookback time ranges analyzed in the main text, and discussed in Fig. 4.2. The results are very similar, with the largest difference occurring for the light fraction of stars younger than $1 \mathrm{Gyr}(\bar{\Delta}=-3.3 \%$ and $\sigma=5 \%)$, and the mass fraction of stars older than $9 \mathrm{Gyr}(\bar{\Delta}=$ $-4.5 \%$ and $\sigma=5.9 \%$ ). These differences explain why the ages obtained with CSP are slightly younger than with SSPs. However, the differences are quite small, and they are smaller than the differences found when comparing results obtained with $G M e$ and $\mathrm{CBe}$ bases, as shown in Fig. B.2 in González Delgado et al. (2015) and Fig. A.1 in González Delgado et al. (2016).
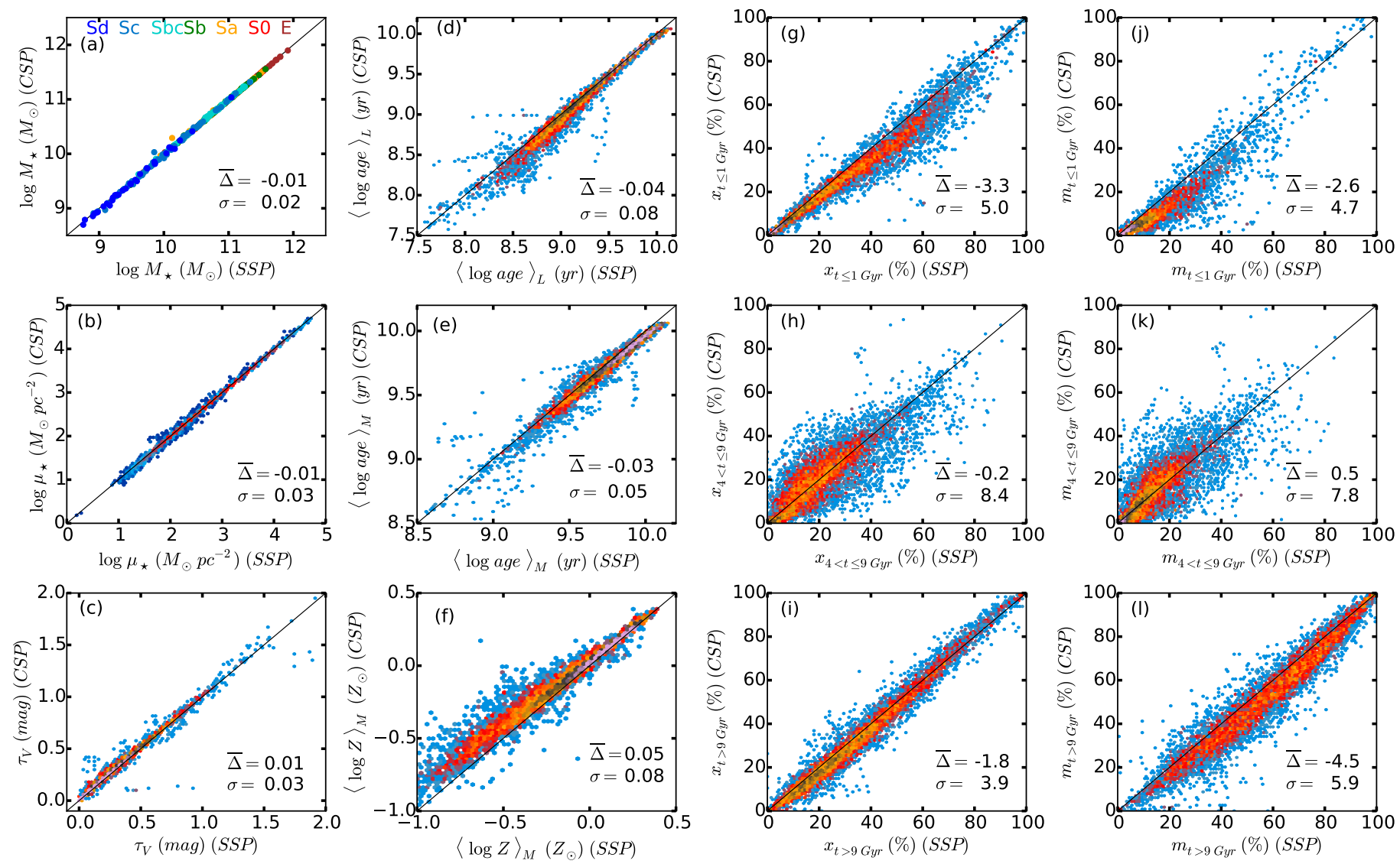

Fig. A.1. Comparison of several stellar population properties as obtained with the CSP ( $y$-axis) and SSP ( $x$-axis) templates. The average difference between the property in the $y$ and $x$-axis is labeled as $\bar{\Delta}$ in each panel, and its standard deviation as $\sigma$. Panel $a$ shows the galaxy mass, with galaxies colored by their Hubble type. In the other panels, the values of the property measured every 0.1 HLR are compared, and the color indicates the density of points in a logarithmic scale (from 0 to 2 ). 